\title{
Simulation of hardening processes, in silicate systems
}

\author{
P. G. Kudryavtsev ${ }^{1, \star}$, O. L. Figovsky ${ }^{2}$ \\ ${ }^{1}$ HIT Holon Institute of Technology, 52 Golomb Street, POB 305 Holon 5810201, Israel \\ ${ }^{2}$ Polymate Ltd - Israel Research Center, POBox 73, Migdal HaEmek 10550, Israel \\ *E-mail address: pgkudr89@gmail.com
}

\begin{abstract}
In this paper we consider the Quasi-homogeneous Approximation to Describe the Properties of Disperse Systems. We have used the statistical polymer method is based on the consideration of averaged structures of all possible macromolecules of the same weight. One has derived equations allowing evaluation of all additive parameters of macromolecules and their systems. The statistical polymer method allows modeling of branched crosslinked macromolecules and their systems in equilibrium or non-equilibrium. The fractal consideration of statistical polymer allows modeling of all kinds of random fractal and other objects studied by fractal theory. The statistical polymer method is applicable not only to polymers but also to composites, gels, associates in polar liquids and other aggregates. In this paper Description of the state of colloidal solutions of silicon oxide from the viewpoint of statistical physics is based on the idea lies in the fact that a colloidal solution of silica silica sol consists of a very large number of interacting with each other particles that are in continuous motion. It is devoted to the study of an idealized system of colliding, but non-interacting particles of sol. Analysis was conducted of the behavior of silica sol, in terms of Maxwell-Boltzmann distribution and was calculated the mean free path of the colloidal particles. Based on these data, it was calculated the number of particles capable to overcome the potential barrier in a collision. To modeling of the solgel transition kinetics had considered various approaches.
\end{abstract}

Keywords: Quasi-homogeneous approximation; statistical polymer method; crosslink formation; fractal method; colloidal solutions; silica sol; sol-gel transition; mean free path

\section{CONTENT}

1. Quasi-homogeneous Approximation to Describe the Properties of Disperse Systems

2. Statistical Polymer Method. Main Notions, Definitions and Equations

3. Crosslink Formation

4. Thermodynamic Functions of Non-Crosslinked Statistical Polymers. Non-Equilibrium

5. Combination of the Statistical Polymer and Fractal Methods

6. Description of the State of Colloidal Solutions of Silicon Oxide from the Viewpoint of Statistical Physics. 
7. Analysis of the Behavior of Silica Sol, in Terms of Maxwell-Boltzmann Distribution

8. The Mean Free Path of the Colloidal Particles

9. Calculation of the Number of Particles Capable to Overcome the Potential Barrier in a Collision.

10. Basic Approaches to Modeling of the Sol-Gel Transition Kinetics

11. Formal Kinetic Description of the Sol-Gel Process

12. CONCLUSION

References

\section{QUASI-HOMOGENEOUS APPROXIMATION TO DESCRIBE THE PROPERTIES OF DISPERSE SYSTEMS}

Lots of real systems are distinguished by extremely irregular geometric structure, which is characterized by a wide range of spatial scales. These systems include - various aerosols, colloidal solutions, suspensions, gas suspension, emulsion, bubble layers, composite materials and porous layers of catalysts, nanocomposites, etc.

Heterogeneity (structural levels) in these systems differs considerably in order of magnitude. For example, in a reactor with a fixed catalyst bed can distinguish at least three characteristic spatial scales: the height of the layer, radius of the grain, the radius of the pores. Determinations of rates of the individual stages of the process observed in such systems are often carried out in the framework of the so-called quasi-homogeneous approximation. It is based on the representation of the inhomogeneous system as a combination of interpenetrating and interacting continuums with effective distributed parameters. Such a representation can be done phenomenologically, based on empirical laws (such as Darcy's law for the filtering process) [1].

Another variant of approach is a hierarchical model building, and is associated with a serial and independent consideration of the process on each of the structural levels. Irregular geometric structure of the space causes a random (fluctuating) the nature of the distribution of temperature, concentrations, reactant streams etc.. Moreover, the range of spatial scales of the fluctuations of the parameters correlated with the spectrum of the characteristic spatial scale of the system. For example, in the case of heterogeneous catalytic reactions, the reactants flows fluctuate on the scale of grain radius due to the random orientation of the pores in the catalyst grains, on the scale of, the pore radius respectively - because the gas filtering features in the spaces between adjacent grains on scale of altitude layer - due to accidental nature of the packing of grains in the layer [2].

With the hierarchical construction of quasi-homogeneous approximation, produce operation averaging (smoothing) fluctuations of the order, it which correspond to the previous small-scale structural level. To do this it is necessary that the characteristic scale of the previous level $\boldsymbol{l}$ was much smaller than the characteristic scale $\boldsymbol{L}$ next level, and the system comprises at the level $\boldsymbol{L}$, macroscopically large number of irregularities of scale $\boldsymbol{l}$. In addition, there must be an intermediate size $\lambda(\boldsymbol{l}<<\lambda<<\boldsymbol{L})$ such that after averaging over the volume $\lambda^{3}$ (or surface $\sim \lambda^{2}$ ) measured parameters $\varphi$, presented not fluctuating, and regular functions of the spatial coordinates, the characteristic scale of changes $\boldsymbol{L}$.

Scale $\lambda$ has the value at which it is much longer than the characteristic distance over which interact fluctuations of scale $\boldsymbol{l}$ and is called the correlation radius. 
Averaging area of size $\lambda$ is called an elementary physical volume or macropoint [3]. For example, the chemical absorption process of gas by liquid in two-phase bubble type reactor, $\boldsymbol{l}$ corresponds to the scale of a gas bubble, a $\boldsymbol{L}$ - reactor size.

Averaging concentrations of the components in each phase is carried out by elementary volume $\lambda^{3}$, containing a sufficiently large number of bubbles, but significantly inferior to the reactor volume. Linear dimension $\lambda$ is chosen taking into account the intensity of the local hydrodynamic mixing. Volume $\lambda^{3}$ regarded as macropoint with efficient (i.e., averaged over the observation time) values of mass transfer coefficients, specific heat release, the distribution of substances between the phases, etc. These parameters are required for the compilation of kinetic equations of individual stages. Then make up the balance equations of mass and energy, taking into account mixing, at a scale of the entire reactor.

In describing the processes in porous materials with liquid or gaseous substances or immiscible liquids, the researchers successfully used a hierarchical approach with the operation of averaging. An example of this approach is to describe the processes such as gas-phase reactions on supported liquid catalysts, catalytic hydrogenation and oxidation of liquid hydrocarbons, electrochemical decomposition of the liquid reactants with the evolution of gaseous products, and drying the impregnated porous materials a multiphase filtering. Under this approach, it was possible to calculate the coefficients of the effective component transport in the porous material, and explain many experimental effects, e.g., removal of the dissolved components on the outer surface, and in macropores during the drying process [4].

Quasi-homogeneous approximation may not be applicable in the study of macrokinetics, of disordered systems, with a wide range of spatial scales, if the correlation radius of the fluctuations, the order of magnitude is comparable to the size of the system L. Such a situation may arise, for example, in chemical reactions in turbulent flow, in a bubbling, and developed fluidized layers.

В этих системах, величина флуктуации скорости и газовый состав, при определенных условиях, достигают размеров устройства. Also, similar situations occur when multiphase processes in porous media, ion exchange membranes and others. First of all, reliable experimental criterion, indicating the presence of large-scale fluctuations, is the emergence of dependence of effective empirical constants on the system size L, in studies of various systems and processes. This phenomenon is observed for the interfacial exchange coefficients and longitudinal diffusion, for fluidized and bubbling layers [5].

For quantitative analysis of strongly fluctuating systems are promising methods developed in the theory of phase transitions of the second kind, and in the study of critical phenomena.

In these systems, near the critical point there are strong fluctuations of the order parameters. In these systems, in the vicinity of the critical point, the experimentally are observed strong fluctuations of the order parameters.

In this paper, we tried to apply the approach quasi-homogeneous approximation to describe the behavior of colloidal solutions such as silica sol. We emphasize that this approach is approximate and may give some deviations in the evaluation of certain parameters. This is due primarily to the nature of the scaling, and the degree of averaging fluctuations.

Also in this method, there are limitations on the number of parameters used, which also contributes to the restriction of accuracy of the estimate of design parameters. However, this method gives a fairly accurate assessment of the direction and nature of the influence of various factors on the properties of the studied systems. 


\section{STATISTICAL POLYMER METHOD. MAIN NOTIONS, DEFINITIONS AND EQUATIONS}

Theoretical description of branched crosslinked polymers, gels and aggregates formed in random processes was traditionally very problematic for polymer science and related disciplines because of difficulties of taking into account all possible structures. Classic methods elaborated by Flory for linear macromolecules [6] are not applicable, in most cases, to branched, especially crosslinked structures [7]. Moreover, if systems of branched crosslinked polymers are in non-equilibrium, their description is impossible without combination of special statistical methods [8,9] and non-linear dynamics of chaos [10].

The problem of modeling of branched cross-linked macromolecules and aggregates was solved recently by the statistical polymer method $[8,9]$.

Let us consider the statistical polymer method in the following order:

(1) modeling of separate macromolecules without crosslinking;

(2) modeling of equilibrium polymeric systems, evaluation of thermodynamic functions, first of all chemical potential;

(3) modeling of complex systems (crosslinking and/or non-equilibrium state, etc.);

(4) possible applications.

\section{Statistical Polymer}

Statistical N-mer is defined as the averaged structure formed by all possible structures of $N$-mers and exhibiting all possible structures ofpolymers containing the same number of monomeric units. In the light of this definition, polymeric systems are considered as sets of assemblages possessing structures averaged on all polymers containing the same numbers of monomeric units - statistical polymers. All interactions in polymeric systems are considered as result of interactions of statistical polymers, and the evaluation of additive (extensive) parameters like energy, entropy etc. may be carried out through statistical polymers instead of branched crosslinked ones.

For large values of A, the number of possible structures is, obviously, much more than the number of monomeric units through the system, and most of the possible structures cannot realize, that could seem to be a drawback of the statistical polymer method. However, if the relaxation time of reactions is much shorter than the measurement duration, one may assume the dynamic equilibrium between various structures that determines correctness of the statistical polymer method even for large enough $N$. Moreover, because of quantum effects the traditional description of macromolecules (as specified structures) is not exact, and this factor of eventual error caused by quantum features of the system allows application of the statistical polymer method for very large macromolecules.

Now, let us consider characteristics of statistical polymer which determine its ability to interact with other statistical polymers and monomers.

Vacancy of the statistical polymer is defined as the capacity of the statistical polymer to capture a monomeric unit. The number of vacancies is denoted as $V(N)$. It is obvious that the capture of an additional monomeric unit by statistical $N$-mer leads to the formation of $(N+1)$ mer.

Extreme unit of the statistical polymer is defined as the monomeric unit which has one only bond with the main structure of the statistical polymer. Let us denote the number of extreme units in statistical $N$-mer as $U(N)$. It is obvious that breaking of the bond of the extreme unit with the main structure of statistical $N$-mer leads to the formation of $(N-1)$-mer. 
Processes of polymerization-destruction in a polymeric system are described as combinations of reactions of statistical polymers:

$$
\begin{gathered}
\operatorname{Pol}(N)+M \Leftrightarrow \operatorname{Pol}(N+1) \\
\operatorname{Pol}(N)+\operatorname{Pol}\left(N_{2}\right) \Leftrightarrow \operatorname{Pol}\left(N_{1}+N_{2}\right)
\end{gathered}
$$

where $\operatorname{Pol}(N)$ is the statistical polymer containing $N$ units; $M$ is a monomer. Since reactions (4.2), in their turn, can be written as combinations of reactions (4.1), these can be considered as independent reactions. Since the process of polymerization is determined by vacancies, whereas destruction - by extreme units, reactions (4.1) can be written in the following form:

$$
\operatorname{Vac}(N)+M \Leftrightarrow \operatorname{Ex}(N+1)
$$

where $E x$ is the extreme unit. If no crosslink forms, the values of $V(N), U(N)$ are given by the following recurrent equations [9]:

$$
\begin{gathered}
V(N+1)=V(N)+m-1=2+N \cdot(m-1) \\
U(N+1)=U(N)+1-\frac{m U(N)}{V(N)}=1+U(N)\left[1-\frac{m}{V(N)}\right]
\end{gathered}
$$

where $m$ is the maximal number of possible branches (functionality minus one).

The kinetics of independent reactions like (4.1) is given by

$$
\left\{\begin{array}{c}
W_{+}(N)=K_{+}[N(m-1)+2] C_{N} C_{1} \\
W_{-}(N)=K_{-} U(N) \cdot C_{N}
\end{array}\right.
$$

where $W_{+}, W_{-}$, and $K_{+}$, are the rates and the rates constants of the direct and the inverse reactions, respectively, and $C_{N}$ is the concentration (mole fraction) of the $N$-mer. For equilibrium,

$$
\begin{aligned}
W_{+}(N)=W_{-}(N+1) & \Rightarrow \frac{C_{N+1}}{C_{1} C_{N}}=\frac{K_{+}[N(m-1)+2]}{K_{-} U(N+1)}=K_{r}(N) \\
K_{+} & =K_{1} \cdot \exp \left(-\frac{E_{a 1}}{R T}\right) \\
K_{-} & =K_{2} \cdot \exp \left(-\frac{E_{a 2}}{R T}\right)
\end{aligned}
$$




$$
K_{r}(N)=K_{0} \cdot \exp \left(-\frac{\Delta E}{R T}\right) \cdot \frac{V(N)}{U(N+1)}
$$

where $T$ - Temperature, $R$ - gas constant;

$K_{0}=\frac{K_{1}}{K_{2}}$ and $\Delta E=E_{a 1}-E_{a 2}$

For large values of $N$, one obtains

$$
\begin{array}{r}
\lim _{N \rightarrow \infty} U(N)=\alpha N, \alpha=\frac{m-1}{2 m-1} \quad(\text { for } \mathrm{M}=1, \mathrm{~m}>1) \\
K_{r}(n \rightarrow \infty)=\frac{K_{+}}{K_{-}}(2 m-1)
\end{array}
$$

The statistical polymer method can be employed also for the description of multicomponent systems, the relevant equations are derived in [8].

\section{CROSSLINK FORMATION}

Let us consider crosslink as the bond between two monomeric units inside the same macromolecule. Hence, crosslink can form only between monomeric units possessing vacancies. Each vacancy can participate in the formation of crosslink with monomeric units which possess vacancies. Hence, the sum number of possibilities of ring formation is

$$
C_{r}=\frac{1}{2} V(N)\left[N-1-B_{0}(N)\right]
$$

where $B_{0}(N)$ is the number of monomeric units which have no vacancies. The value of $B_{0}(N)$ can be estimated from

$$
B_{0}(N+1)=B_{0}(N)+\frac{B_{1}(N)}{V(N)}
$$

taking into account that for monomeric units with $s$ vacancies $(1 \leq s \leq(m-1))$ :

$$
\begin{gathered}
B_{s}(N+1)=B_{s}(N)+(s+1) \frac{B_{(s+1)}(N)}{V(N)}-s \frac{B_{S}(N)}{V(N)} \\
B_{m} \equiv U(N)
\end{gathered}
$$

The rate of reaction of crosslink formation is 


$$
W_{c+}=K_{c+} C_{r}
$$

The rate of reaction of crosslink destruction is

$$
W_{c-}=K_{c-} G_{r}
$$

where $G_{r}(N)$ is the number of crosslinks, whereas $K_{c^{+}}$and $K_{c-}$ are the constants of crosslink formation and destruction, respectively.

In equilibrium:

$$
\begin{gathered}
W_{c+}=W_{c-} \Rightarrow K_{c+} C_{r}=K_{c-} G_{r} \\
K_{c r}=\frac{K_{c+}}{K_{c-}}=\frac{G_{r}}{C_{r}}=\frac{2 G_{r}}{V(N)\left[N-B_{0}(N)-1\right]}
\end{gathered}
$$

The crosslink formation reduces the number of vacancies and extreme units:

$$
\begin{gathered}
V^{\prime}(N)=(m-1) N+2-2 G_{r} \\
U^{(c+1)}(N)=U^{(c)}(N)-m U^{(c)}(N) \frac{G_{r}(N)}{V(N)}
\end{gathered}
$$

or

$$
U(l)=U^{0} \prod_{k=1}^{l}\left[1-\frac{2 m}{V^{0}-2(k-1)}\right]
$$

where index " 0 " means the non-crosslinked state, $l$ - the number of crosslinks.

The ring formation reduces both the number of vacancies and that of extreme units; therefore one may assume that the weak ring formation does not significantly influence the weight distribution of polymers.

\section{THERMODYNAMIC FUNCTIONS OF NON-CROSSLINKED STATISTICAL POLIMERS. NON-EQUILIBRIUM}

From Eq. (4.10) one obtains

$$
\frac{\Delta S^{0}}{R T}=\frac{\Delta H^{0}}{R T}+\ln K_{0}-\frac{\Delta E_{a}}{R T}+\ln V_{\Sigma}(N)-\ln U_{\Sigma}(N+1)
$$

However, the heat effect of a reaction of polymerization

$$
\Delta H^{0}=\Delta E_{a}=2 \varepsilon_{0}
$$


where $\varepsilon_{0}$ is the energy of a vacancy (all vacancies are assumed to be equivalent!). From Eqs. (4.25) and (4.26) one obtains

$$
\Delta S^{0}(N)=R\left[\ln K_{0}+V_{\Sigma}(N)-\ln U_{\Sigma}(N+1)\right]
$$

However

$$
\Delta S^{0}(1)=R\left[\ln K_{0}+\ln V_{\Sigma}(1)-\ln U_{\Sigma}(2)\right]
$$

Taking into account that $V_{\Sigma}(1)=(m+1)$, and $U_{\Sigma}(2)=2$ [9], one obtains

$$
\begin{gathered}
\ln K_{0}=\frac{\Delta S^{0}(1)}{R}+\ln \frac{2}{m+1} \\
\Delta S^{0}(N)=\Delta S^{0}(1)+R \ln \frac{2 V_{\Sigma}(N)}{(m+1) U_{\Sigma}(N+1)}
\end{gathered}
$$

The chemical potential of statistical $N$-mer is given by

$$
\mu^{0}(N)=\varepsilon_{0} V_{\Sigma}(N)-T\left[N S^{0}(1)+N \Delta S^{0}(1)+R \sum_{n=1}^{N} \ln \frac{2 V_{\Sigma}(n-1)}{(m+1) U_{\Sigma}(n)}\right]
$$

where $C_{N}$ is the concentration of $N$-mer; $S^{0}(1)$ - the entropy of monomer.

Now, let us consider a non-equilibrium chemical process in a polymeric system described in linear approximation:

$$
\left\{C_{1 n}\right\} \rightarrow\left\{C_{2 n}\right\}
$$

where indexes " 1 " and " 2 " correspond to the initial and final states, respectively. The Gibbs' energy in these states is given by

$$
\begin{aligned}
\Delta G_{1} & =\sum_{n=1}^{\infty}\left(\mu_{1 n}^{0}+R T \ln C_{1 n}\right) C_{1 n} \\
\Delta G_{12} & =\sum_{n=1}^{\infty}\left(\mu_{2 n}^{0}+R T \ln C_{2 n}\right) C_{2 n}
\end{aligned}
$$

The moving force of the process (32) is 


$$
\Delta G=\sum_{n=1}^{\infty}\left(\mu_{n}^{0}+R T \ln C_{n}\right) C_{n}=\sum_{n=1}^{\infty} \mu_{n} C_{n}=\sum_{n=1}^{\infty} \Omega_{n}
$$

where $\Omega_{n}=\mu_{n} C_{n}$.

Description of non-linear situations can be carried out in the same style, using methods of dynamic of chaos [10].

\section{COMBINATION OF THE STATISTICAL POLYMER AND FRACTAL METHODS}

Let us consider statistical $N$-mer $(N \rightarrow \infty)$ as the fractal with dimensionality $D_{f}$. Such approach can be compared to the accepted practice of the construction of fractal clusters by the Monte-Carlo method of random addition of new units; only difference - the statistical polymer is automatically random and contains all possible structures of randomly constructed clusters (of course, if they contain the same number A of units). We may assume that the statistical $\mathrm{N}$ mer can be considered as the averaged structure obtained after the infinite number of operations of constructions of $N$-meric clusters.

We note some obvious advantages of the statistical polymer approach in comparison with the Monte-Carlo method of the construction of cluster: (1) Monte-Carlo method is not strictly random, that causes several errors which are reduced and disappear only for $\infty$-meric clusters; (2) the application of the Monte-Carlo method to the 3-dimensional systems is too difficult, whereas the statistical polymer method is applicable in all situations, that offers much more freedom to the researcher; (3) the Monte-Carlo method furnishes numerical results, whereas the statistical polymer method allows the obtainment of analytical ones (at least, for all additive parameters of macromolecules and polymeric systems); (4) for the obtainment of the same result, the Monte-Carlo method requires much more calculations.

Since we consider statistical $N$-mers (at very large $N$ ) as random fractal-like objects, we need to define the characteristic dimension (size) of the fractal statistical polymer. Let us define the characteristic size of fractal statistical $N$-mer (at very large $N$ ) as follows:

$$
\begin{gathered}
Z(N)=d_{0} \sum_{k=1}^{N} W_{k}(N) \\
W_{k}(N)=\left\{\begin{array}{cc}
1 & \text { if } R_{k}(N) \geq 1 \\
R_{k}(N) & \text { for } R_{k}(N)<1
\end{array}\right.
\end{gathered}
$$

where $d_{0}$ is the characteristic size of monomeric unit $\left(d_{0}=\sqrt[3]{v_{0}}, v_{0}\right.$ is the volume of monomer unit), and the parameter $R_{k}(N)$ (presence) characterizes the distribution of monomeric units inside the statis- deal polymer [9]. Since the validity of the fractal approach is assumed, the volume and the surface area of such $N$-mer are given by

$$
V_{f}=V(N) \sim[Z(N)]^{D_{f}}
$$




$$
A_{f}=A(N) \sim[Z(N)]^{D_{f-1}}
$$

where $D_{f}$ is the fractal dimensionality.

On the other hand, the same parameters are easily estimated from the total number of monomeric units:

$$
\begin{gathered}
V_{p}=V(N)=N v_{0} \\
A_{p}=A(N)=N a_{0}=\alpha_{s} N\left(v_{0}\right)^{2 / 3}
\end{gathered}
$$

where $a_{0}$ is the surface area per monomeric unit, and as a normalization coefficient.

According to assumptions made above, Eqs. (4.36)-(4.38) should be correct at very large $N$, while for low and moderate values of $N$, they should not. This means that the total divergence between the values obtained from Eqs. (4.37)-(4.40) is large at small $N$ and reduces at infinite $N$, that is characterized by the following functional ref. [8]:

$$
\begin{aligned}
& \Phi_{v}=\Phi_{v}\left(N_{1}, N_{2}\right)=\frac{1}{4\left(N_{2}-N_{1}+1\right)} \times \sum_{N=N_{1}}^{N_{2}}\left[\frac{V_{f}(N)}{V_{p}(N)}-\frac{V_{p}(N)}{V_{f}(N)}\right]^{2} \\
& \Phi_{A}=\Phi_{A}\left(N_{1}, N_{2}\right)=\frac{1}{4\left(N_{2}-N_{1}+1\right)} \times \sum_{N=N_{1}}^{N_{2}}\left[\frac{A_{f}(N)}{A_{p}(N)}-\frac{A_{p}(N)}{A_{f}(N)}\right]^{2}
\end{aligned}
$$

Functional (4.41) was applied to the computer treatment of Eqs. (4.36), (4.37), (4.39) at various $\mathrm{m}$, with minimization of the functional (4.41); the varied parameter of optimization was $D_{f}$.

The general correlation between values of $V(N)$ estimated from Eqs. (4.36), (4.39) at $m=3, N_{1}=500, N_{2}=2000$, is presented in Figure 1.

We see that the relative divergence between curves $V_{f}(N)$ and $V_{p}(N)$ is very large at small $N$, but decreases at large $N$.

The relative square dispersion is $\left(\Phi_{v}\right)_{\min }=0.052$ (relative error about 0.24 ) and is caused mostly by the initial parts of both curves, that is enough good for the correlation with single optimization parameter.

In this case, the fractal dimensionality $D_{f}=2.32$.

In the general case, of course, the fractal dimensionality depends on $m$ :

$$
\begin{aligned}
& \lim _{m \rightarrow 1} D_{f}=1 \\
& \lim _{m \rightarrow \infty} D_{f}=3
\end{aligned}
$$




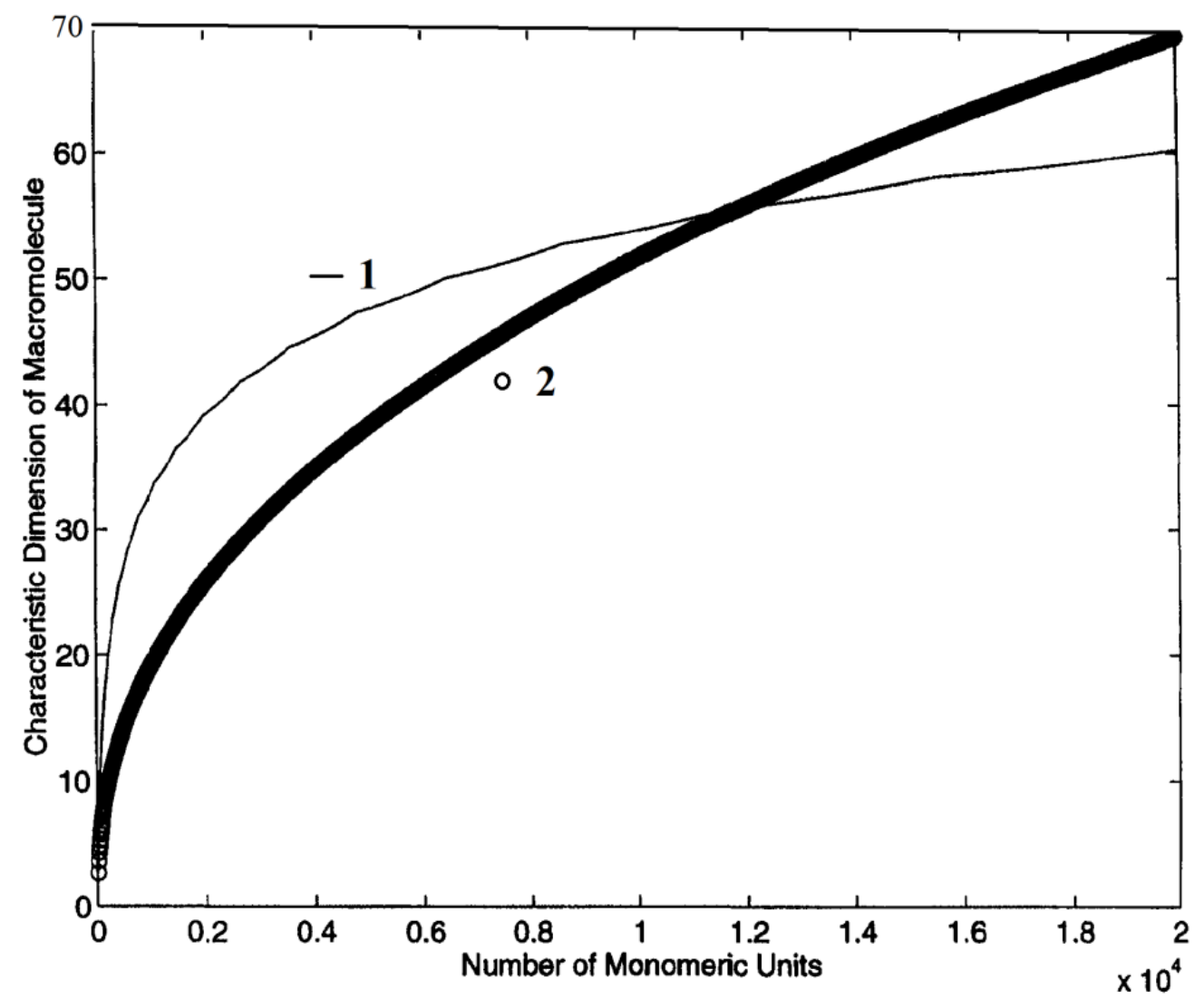

Figure 1. Dimension of Macromolecule: Statistical Polymer (1) and Fractal Approach (2).

\section{Applicability of the statistical polymer method}

The statistical polymer method allows modeling of not only polymers but also gels, several composites, associates in polar liquids, etc.

The combined fractal- statistical polymer method is applicable to all objects described by fractal theory, first of all random fractals.

The statistical polymer method can be very effective for the solution of numerous problems of materials science, because many of widespread materials exhibit macromoleculelike fragments. However, in such cases one should take into account that there are two kinds of interactions: inside macromolecule-like fragments ("strong" interactions) and between them ("weak" interactions). "Weak" interactions determine first of all mechanical stability, permeability and sometimes adhesion properties of the material.

The statistical polymer method is based on the consideration of averaged structures of all possible macromolecules of the same weight. One has derived equations allowing evaluation of all additive parameters of macromolecules and their systems. The statistical polymer method allows modeling of branched crosslinked macromolecules and their systems in equilibrium or non-equilibrium. The fractal consideration of statistical polymer allows modeling of all kinds of random fractal and other objects studied by fractal theory. The statistical polymer method is applicable not only to polymers but also composites, gels, assonates in polar liquids and other aggregates. 


\section{DESCRIPTION OF THE STATE OF COLLOIDAL SOLUTIONS OF SILICON OXIDE FROM THE VIEWPOINT OF STATISTICAL PHYSICS}

This section is based on the idea lies in the fact that a colloidal solution of silica - silica sol consists of a very large number of interacting with each other particles that are in continuous motion.

It is devoted to the study of an idealized system of colliding, but non-interacting particles of sol. To simplify the approach, consider the ideal system of monodisperse colloidal particles. Since the sol particles much larger than the molecules of the solvent in which they are located, their interactions with solvent molecules can be neglected and consider this system as a suspension of the sol particles in a certain continuous environment - continuum. Sol particles most of the time are running freely and do not collide with each other. They interact only during collisions, causing the speed and direction of movement, each of the colliding particles varies. Therefore, trajectory of motion of particles in the sol is a broken line.

With certain assumptions, the ideal gas laws can be applied to such a system. Consider a system of $\boldsymbol{N}$ particles filling a certain volume of space $\boldsymbol{V}$. The distribution of particles in space is described by the function:

$$
C_{n}=C_{n}(t, \vec{r})
$$

depending on the time and coordinates. This function is called countable concentration and is defined as follows. We partition the space filled with sol particles, into small pieces and look at one of these parts, the volume of which we denote by $d \boldsymbol{V}$. Volume $d \boldsymbol{V}$ called physically infinitely small if the number of $d \boldsymbol{N}$ of particles therein substantially smaller than the total number $\boldsymbol{N}$ of particles in volume $\boldsymbol{V}$, but in this case, many times greater than unity: $1 \ll d N \ll$ $N$. The position of volume $d \boldsymbol{V}$ in space can be set using the radius vector $\vec{r}$ one of its points. The ratio number $d \boldsymbol{N}$ of particles in the volume $d \boldsymbol{V}$ to the value of this volume, called a countable particle concentration, in the area of space:

$$
C_{n}(t, \vec{r})=\frac{d N}{d V}
$$

Since in its motion particles can enter into the volume $d \boldsymbol{V}$ and out of it, the number of particles $d \boldsymbol{N}$, in this volume, strictly speaking, will change over time. Therefore, the concentration is a function not only of the radius vector $\vec{r}$, but also the time $t$. By integrating the equation (4.44), we obtain the number $N$, equal to the number of particles in the volume $V$ :

$$
N=\int_{V} C_{n}(t, \vec{r}) d V
$$

When the particles are distributed throughout the volume of the vessel, on average uniformly whilst their concentration everywhere is identical, and equation (4.45) becomes: 


$$
N=C_{n} \cdot V
$$

The physical meaning of countable concentration can be defined as the number of particles per unit volume. In fact, putting $\boldsymbol{V}=1 \mathrm{~m}^{3}$, we obtain $\boldsymbol{C}_{\boldsymbol{n}}=\boldsymbol{N}$.

Let us consider the probability that a randomly selected one of sol particle in time $\boldsymbol{t}$ will be in a volume $d \boldsymbol{V}$ :

$$
d W=\frac{d N}{N}
$$

Thus it is possible to get a value of the probability density:

$$
w(t, \vec{r})=\frac{d W}{d V}
$$

Probability density, also called the distribution function, of the sol particles in space. This function is related to the countable concentration of sol, the following simple equation:

$$
C_{n}(t, \vec{r})=N w(t, \vec{r})
$$

For the case of monodisperse particles, can obtain the equation for the mass concentration of particles in a unit volume of sol:

$$
C(t, \vec{r})=m_{p} C_{n}(t, \vec{r})
$$

Where: $\boldsymbol{m}_{\boldsymbol{p}}$ - the mass of one particle of sol. For the spherical sol particles with a diameter $\boldsymbol{D}_{\boldsymbol{p}}$, when of sol substance density $\boldsymbol{\rho}$, it is equal to:

$$
m_{p}=V_{p} \rho=\frac{\pi D_{p}^{3}}{6} \rho
$$

In the case of polydisperse systems, it is necessary to introduce a new function for a countable concentration which also depends on the mass of particles of the appropriate size $C_{n m}\left(t, m_{p}, \vec{r}\right)$. Accordingly, the expression for the mass concentration will be:

$$
C_{n}(t, \vec{r})=\int_{0}^{\infty} \varphi\left(m_{p}\right) C_{n m}\left(t, m_{p}, \vec{r}\right) d m_{p}
$$

Where: $\varphi\left(m_{p}\right)$ - distribution function of particle mass in the sol. 
However, to simplify the description and analysis of the system of the sol particles we consider the simplified case of a monodisperse of sol.

To describe the microscopic state of the sol, we use the function:

$$
f=f(t, \vec{r}, \vec{v})
$$

This function depends on the time $t$, the radius vector $\vec{r}$ and the speed vector $\vec{v}$, and called the distribution function. It is defined as follows. Let us consider a particle that at time $t$ were in the volume $d \boldsymbol{V}$ at the radius vector $\vec{r}$. The number of such particles is $-d \boldsymbol{N}$. The movement of each particle is characterized by a certain speed vector $\vec{v}_{i}$, where $\boldsymbol{i}$ - number of the particle.

We construct an imaginary rectangular coordinate system, on the axes of which we plot the values of projections $v_{x}, v_{y}$ and $v_{z}$ of the velocity vector of each particle in this volume. Space formed by using this coordinate system, called the space of velocities. Single out small volume in the velocity space, which is physically infinitesimal, the value of this volume is denoted $d^{3} v$. Speed of some particles from the $d \boldsymbol{N}$ end in this volume: $\overrightarrow{v_{1}} \in d^{3} v$. Let their number be $d N^{\prime}$. And this value satisfies the inequalities $1 \ll d N^{\prime} \ll d N$. The distribution function (4.53) is defined as the ratio of the number of particles $d N^{\prime}$, to the product of volumes of $d \boldsymbol{V}$ and $d^{3} v$ :

$$
f(t, \vec{r}, \vec{v})=\frac{d N^{\prime}}{d V d^{3} v}
$$

From this definition it follows that the number of particles $d N^{\prime}$, which at time $t$, appeared in volume $\boldsymbol{d} \boldsymbol{V}$, with the radius vector $\vec{r}$, and their velocity vectors ending in the volume of $d^{3} v$, with the vector $\vec{v}$, can be calculated if we know the distribution function (4.53). Separating the variables and integrating the equation (4.54) in terms of volume, and then dividing it by $d \boldsymbol{V}$, and considering that the integration is performed over the velocity space, we obtain the equation:

$$
C_{n}(t, \vec{r})=\int f(t, \vec{r}, \vec{v}) d^{3} v
$$

This equation relates the concentration of the particles and their distribution function. The value of the elementary volume $d^{3} v$ in velocity space can be represented as the product of differentials of the projections of the velocity vector $\vec{v}$ on the coordinate axes, which makes the integral (4.55) in the triple integral:

$$
C_{n}(t, x, y, z)=\int_{-\infty}^{+\infty} \int_{-\infty}^{+\infty} \int_{-\infty}^{+\infty} f\left(t, x, y, z, v_{x}, v_{y}, v_{z}\right) d v_{x} d v_{y} d v_{z}
$$

Where: integration is performed over all possible values of variables $v_{x}, v_{y}$ and $v_{z}$. 
According to the definition (4.47), we can determine the probability that one randomly selected particle of the sol at time $\boldsymbol{t}$ stored in the volume $d \boldsymbol{V}$, and its velocity - in the volume $d^{3} v$ of space velocity. The corresponding probability density is defined as the ratio

$$
w(t, \vec{r}, \vec{v})=\frac{d W}{d V d^{3} v}
$$

As follows from formula (4.54), the probability density associated with the distribution function (4.53) by the relation

$$
f(t, \vec{r}, \vec{v})=N w(t, \vec{r}, \vec{v})
$$

Particle velocity distribution is isotropic, if the distribution function depends only on the modulus $v$ of the velocity vector $\vec{v}$, and does not depend on its direction: $f=f(t, \vec{r}, v)$. With such velocity distribution of particles, the particle fluxes moving in different directions are the same. In other words, all the directions of particle motion in the space are equal. In this case, the average velocity of the directed motion of the particles obviously must be equal to zero: $\vec{u}=0$. This means that the direction of motion of the particles is absent. This situation is typical in cases where the system does when mixing and sol particles are small enough, and the effect of sedimentation of particles under the influence of gravity can be neglected.

An important factor in determining the behavior of the sol is the average velocity of the of the sol particles. To determine them, we assume that $d \boldsymbol{N}$ is the number of particles in the volume $d \boldsymbol{V}$. We find the average value of $\bar{v}$ module $v$ of velocity vector of particles. For this purpose, it is necessary to sum the modules of velocities of particles in a volume $d \boldsymbol{V}$, and the resulting sum divide into the number of particles:

$$
\bar{v}=\frac{1}{d N} \sum_{i} v_{i}
$$

where the index $\boldsymbol{i}$ denotes the number of particles in a volume $d \boldsymbol{V}$. As the volume of $d^{3} v$ in velocity space sufficiently small for those particles whose velocity, end up in this volume, we can put $\vec{v}_{i}=\vec{v}$ with $\vec{v}_{i} \in d^{3} v$, where $\vec{v}$ - arbitrary vector ending in volume $d^{3} v$. Therefore, the sum of modules velocities of the particles is equal to the product of the module $v$ velocity of one particle on the number $d N^{\prime}$ :

$$
v d N^{\prime}=v f(t, \vec{r}, \vec{v}) d V d^{3} v
$$

Sum of modules velocities of all particles in the volume $d \boldsymbol{V}$ will be equal to the integral over velocity space of this expression:

$$
\sum_{i} v_{i}=d V \int v f(t, \vec{r}, \vec{v}) d^{3} v
$$


Substituting of this sum in (4.59) with (4.44) yields

$$
\bar{v}=\frac{1}{C_{n}} \int v f(t, \vec{r}, \vec{v}) d^{3} v
$$

Actually, this equation represents the moment of the 1 st order of the function of threedimensional probability distributions. Similarly, we can derive formulas for the moments of other orders of the probability distribution function. For example, the mean values of $\overline{\vec{v}}$ and $\overline{v^{2}}$ of the velocity vector and the square of its module, we have the following formulas

$$
\begin{gathered}
\overline{\vec{v}} \equiv \vec{u}(t, \vec{r})=\frac{1}{C_{n}} \int \vec{v} f(t, \vec{r}, \vec{v}) d^{3} v \\
\overline{v^{2}}=\frac{1}{C_{n}} \int v^{2} f(t, \vec{r}, \vec{v}) d^{3} v
\end{gathered}
$$

In the general case, the vector $\vec{u}$ average particle velocity is not zero and is a function of time and the radius vector. This means that at any given time in a given place of space, the whole weight of the sol, as a whole moves in a certain direction. Figuratively speaking, sol flows along the guide, or it is stirred in the reaction vessel. In solving some problems it is convenient to assume that the sol particles are involved in two movements: the random thermal motion and direction of motion, which is characterized by a vector of average speed $\vec{u}$.

Note that the integration in (4.60)-(4.62) is performed over the space of velocities for given values of $\boldsymbol{t}$ and $\vec{r}$. Therefore, the expressions obtained after integration generally will represent some functions of the time and the coordinates.

Value

$$
v_{R M S} \equiv\langle v\rangle=\sqrt{\overline{v^{2}}}
$$

called the mean square velocity of the particle.

\section{ANALYSIS OF THE BEHAVIOR OF SILICA SOL, IN TERMS OF MAXWELL-BOLTZMANN DISTRIBUTION}

Let us consider silica sol, which is in a state of thermodynamic quasi-equilibrium. Function (4.11), which describes the distribution of the sol particles in space and velocity, can be found using the Gibbs canonical distribution. For this should be considered a colloidal solution, as an ensemble, each of sol particles, it is one of the systems. The parameter in the canonical Gibbs distribution, should be understood as an aggregate of the spatial coordinates $x$, $y, z$, and projections $v_{x}, v_{y}, v_{z}$ of the velocity vector of the particle:

$$
x \equiv\left\{x, y, z, v_{x}, v_{y}, v_{z}\right\} \equiv\{\vec{r}, \vec{v}\}
$$


According to the law Gibbs probability density for a particle in the ideal gas approximation for a system in a state of thermodynamic equilibrium or near-equilibrium state will have the form

$$
w(\vec{r}, \vec{v})=v \exp (-\beta \varepsilon(\vec{r}, \vec{v}))
$$

Where $\beta=(k T)^{-1}$ - the inverse temperature, and

$$
\varepsilon(\vec{r}, \vec{v})=\frac{1}{2} m v^{2}+U(\vec{r})
$$

$\varepsilon(\vec{r}, \vec{v})$ - That part of energy of the particle that depends on its velocity and coordinates, i.e. the sum of the kinetic energy of the translational motion of a particle and its potential energy. This equation is valid if the system has an external field, of conservative force (for example, if the force of gravity acting on the particles). Besides the energy, as described in equation (4.64), the particle also has internal energy, which, in this situation, has no significant effect on the properties of an ensemble of particles in terms of their interaction with each other.

Knowing the probability density (4.63), we find the distribution function by the formula (4.58):

$$
f(\vec{r}, \vec{v})=N v \exp \left\{-\beta\left(\frac{1}{2} m v^{2}+U(\vec{r})\right)\right\}
$$

Function (4.63) and (4.64) are called distributions Maxwell - Boltzmann. One of the characteristics of these functions is that they depend only on the modulus of the velocity vector and does not depend on the direction in space. In other words, these functions describe the isotropic velocity distribution of particles.

Distribution function (4.64) is conveniently represented as a product of two functions:

$$
f(\vec{r}, \vec{v})=n(\vec{r}) \cdot w(\vec{v})
$$

The first of these is the concentration of particles:

$$
n(\vec{r})=n_{0} \exp (-\beta U(\vec{r}))
$$

The second is the probability density:

$$
w(\vec{v})=\mathrm{A} \exp \left(-\alpha v^{2}\right)
$$

Here, A - normalization constant, and $\alpha$ - is: 


$$
\alpha=\frac{\beta m}{2}=\frac{m}{2 k T}
$$

Constants $\boldsymbol{n}_{\mathbf{0}}$ and A connected by the relation:

$$
n_{0} A=N v
$$

Function (4.66) is called the Boltzmann distribution and the function (4.67) - Maxwell distribution. The first describes the distribution of particles in space, and the second - the velocity distribution.

Substituting the function (4.65) into (4.55) leads to the normalization condition for the function (4.67):

$$
\int w(\vec{v}) d^{3} v=1
$$

Expanding the integral (4.69) along the axes of the particle velocity vector and using the expression for the Poisson integral, we find the normalization constant:

$$
A=\left(\frac{\alpha}{\pi}\right)^{\frac{3}{2}}
$$

Using the expression (4.70) for the normalization constant, we write the Maxwell distribution (4.67) as follows:

$$
w(\vec{v})=\left(\frac{\alpha}{\pi}\right)^{\frac{3}{2}} \exp \left(-\alpha v^{2}\right)
$$

This function can be represented as the product of three functions:

$$
w(\vec{v})=g\left(v_{x}\right) g\left(v_{y}\right) g\left(v_{z}\right)
$$

Where

$$
g\left(v_{x}\right)=\left(\frac{\alpha}{\pi}\right)^{\frac{1}{2}} \exp \left(-\alpha v_{x}^{2}\right)
$$

a so-called Gaussian function.

Calculate the mean velocity $u$ directed motion particles of the sol, by the formula for the first moment of the distribution function, which is the distribution function Maxwell Boltzmann takes the form: 


$$
\vec{u}=\overrightarrow{\vec{v}}=\int \vec{v} w(\vec{v}) d^{3} v
$$

Using the function (4.71), we obtain the following expression for the average value of the projection of the velocity vector on the $x$-axis:

$$
u_{x} \equiv \bar{v}_{x}=\int_{-\infty}^{+\infty} v_{x} g\left(v_{x}\right) d v_{x} \int_{-\infty}^{+\infty} g\left(v_{x}\right) d v_{x} \int_{-\infty}^{+\infty} g\left(v_{x}\right) d v_{x}
$$

As might be expected, for an isotropic particle velocity distribution, this expression is zero, because the integral of an odd function is zero $v_{x} g\left(v_{x}\right)$. Thus for particles whose velocities are have Maxwellian distribution, the average speed of the directional motion is zero: $\vec{u}=0$.

From this, the mean square of the projection of the velocity vector on the x-axis, is:

$$
\overline{v_{x}^{2}}=\int v_{x}^{2} w(\vec{v}) d^{3} v
$$

If we expand this integral along the axes of the space velocities of the particles, and substituting in it the expression (4.71), we obtain:

$$
\overline{v_{x}^{2}}=\int_{-\infty}^{+\infty} v_{x}^{2} g\left(v_{x}\right) d v_{x} \int_{-\infty}^{+\infty} g\left(v_{x}\right) d v_{x} \int_{-\infty}^{+\infty} g\left(v_{x}\right) d v_{x}
$$

Integration of this expression gives the formula:

$$
\overline{v_{x}^{2}}=\frac{k T}{m}
$$

Because of the properties of random variables, the mean value of the sum of two random variables is the sum of the average values of these variables; the mean square of the velocity modulus is equal to:

$$
\overline{v^{2}}=\overline{\left(v_{x}^{2}+v_{y}^{2}+v_{z}^{2}\right)}=\overline{v_{x}^{2}}+\overline{v_{y}^{2}}+\overline{v_{z}^{2}}
$$

The equilibrium distribution function (4.26) depends on the modulus of the velocity vector does not depend on its direction, i.e. it describes an isotropic velocity distribution of particles, all directions are equal. Therefore, the mean square of the projection of the velocity vector on any axis does not depend on the direction of this axis in space:

$$
\overline{v_{x}^{2}}=\overline{v_{y}^{2}}=\overline{v_{z}^{2}}
$$


Wherein

$$
\overline{v^{2}}=3 \overline{v_{x}^{2}}
$$

and in view of (4.37) (4.74), we have:

$$
\overline{v^{2}}=\frac{3 k T}{m}
$$

Using the definition (4.63), we find the mean square velocity of the particle:

$$
\langle v\rangle=\sqrt{\frac{3 k T}{m}}
$$

Since the Maxwell distribution function of (4.67) depends on the modulus of the velocity vector, and does not depend on its direction, in this connection, on the sphere radius $v$ in the space of velocity, this function everywhere takes the same value.

The number $d \boldsymbol{N}^{\prime}$ of particles in volume $d \boldsymbol{V}$, the velocity modulus which belong to the interval $(v, v+d v)$ can be found from the formula (4.54). For this purpose we substitute in this formula the function (4.65) and the volume

$$
d^{3} v=4 \pi v^{2} d v
$$

of spherical layer

$$
d N^{\prime}=n w(\vec{v}) d V \cdot 4 \pi v^{2} d v
$$

Value

$$
d N=n d V
$$

is the number of particles in the volume $d \boldsymbol{V}$. By definition, the ratio of

$$
\frac{d N^{\prime}}{d N}=w(\vec{v}) 4 \pi v^{2} d v
$$

there is a possibility that one of the particles has a velocity whose modulus is in the range from $v$ to $v+d v$.

We introduce the function $F=F(v)$, depending on the modulus of the vector velocity by the relation

$$
F(v) d v \equiv w(\vec{v}) 4 \pi v^{2} d v
$$

Using the expression (4.34), we have 


$$
F(v)=4 \pi\left(\frac{\alpha}{\pi}\right)^{\frac{3}{2}} v^{2} \exp \left(-\alpha v^{2}\right)
$$

Equation (4.79) is called, the Maxwell function. For $v=0$, the function (4.79) is equal to zero: $F(0)=0$. If the value $v_{b}$ of speed module, which is called the most probable velocity of the particle, Maxwell functions has a maximum. In the interval $\left(0, v_{b}\right)$ it is monotonically increasing, and in the interval $\left(v_{b}, \infty\right)$ it is monotone decreasing, tends to zero as $v \rightarrow \infty$.

Because the expression $F(v) d v$ represents the probability respectively integral of this expression is equal to one:

$$
\int_{0}^{\infty} F(v) d v=1
$$

The physical meaning of the function Maxwell relations for sol can be explained as follows. According to the definition of probability expression $F(v) d v$ is the fraction of particles, modules velocities are in the range of $(v, v+d v)$. The relative number of particles whose velocities are in the range of $v_{1}$ to $v_{-} v_{2}$, will be expressed by the integral

$$
\frac{N\left\{v \in\left[v_{1}, v_{2}\right]\right\}}{N}=\int_{v_{1}}^{v_{2}} F(v) d v
$$

where $N$ - the total number of particles considered, $N\left\{v \in\left[v_{1}, v_{2}\right]\right\}$ - number of particles, modules velocities are in the range $\left[v_{1}, v_{2}\right]$. Let us find the most probable velocity $v_{b}$ of the particles. The necessary condition for an extremum function, the derivative of $F=F(v)$ for this value is zero. Equating to zero the derivative with respect to the parameter $v$, from the expression (4.79) we find required value of the most probable velocity of the particles, which is equal to:

$$
v_{b}=\frac{1}{\sqrt{\alpha}}=\sqrt{\frac{2 k T}{m}}
$$

Substituting this value into the formula (4.79), we obtain the maximum value of the Maxwell function

$$
F_{\max }=F\left(v_{b}\right)=\frac{4}{e} \sqrt{\frac{\alpha}{\pi}}=\frac{4}{e} \sqrt{\frac{m}{2 \pi k T}}
$$

Maxwell function (4.79) contains as a parameter value $\alpha$, which according to formula (4.68) depends on the temperature of the sol. Therefore, the Maxwell function change when there is a change in temperature of the sol. The same is a change in the particle velocity 
distribution. The most probable velocity of particles (4.80) increases with increasing temperature. Thus, the maximum value of the Maxwell function (4.82) decreases with an increasing temperature. The form of the graphic depending of the Maxwell function with increasing temperature is altered so that the maximum of the curve shifts to the right (towards high velocities) and becomes lower, but the area under the curve, however, remains equal to unity.

We will analyze how changes with temperature, the particle velocity distribution. For this purpose we choose some arbitrary value of velocity $v_{0}$. The relative number of particles $N\left(v<v_{0}\right) / N$ and $N\left(v>v_{0}\right) / N$ at speeds respectively, smaller and larger than $v_{0}$, expressed by integrals of Maxwell functions:

$$
\frac{N\left(v<v_{0}\right)}{N}=\int_{0}^{v_{0}} F(v) d v
$$

and

$$
\frac{N\left(v>v_{0}\right)}{N}=\int_{v_{0}}^{\infty} F(v) d v
$$

It is easy to see that with increase in temperature the amount of $N\left(v<v_{0}\right)$ particles with velocities $v<v_{0}$ monotonically decreases, and the amount of $N\left(v>v_{0}\right)$ particles with velocities $v>v_{0}$ increases.

This property is allows us to calculate the number of particles with velocities above a certain level, and thus determine the number of particles that are able to interact with each other or with other objects if necessary to overcome some potential barriers.

\section{THE MEAN FREE PATH OF THE COLLOIDAL PARTICLES}

Colloidal particles in a solution are in continuous motion. Consider first approximation model colloidal particle motion in the approximation of an ideal gas. We believe that the solvent is a continuum, does not affect the nature of the interaction between the particles. The particles can be represented in the form of small solid elastic spheres of diameter $d$, the collision of which there is no interaction. The entire system is also represented in the form of an infinitely large vessel, the walls of which have no effect on the behavior of colloidal particles in the system. All particles in the system have the same size.

Each particle moves uniformly and rectilinearly, as long as does not collide with any other particle. As a result of the collision, the particle abruptly changes its direction of motion, and then again moves in a straight line at a constant speed until the next collision.

Using this model, we calculate the average time between two successive collisions of one arbitrarily selected particle with other particles of the colloidal solution. Selected particle collision with another particle will occur only when center of the particle, would be at the moment of convergence between the particles at a distance from the line of movement of the first particle less than particle diameter d (Figure 2). Therefore, the centers of all the particles 
encountered on line trajectory selected particle, for some time $\Delta \mathrm{t}$, and forcing it to change its direction of motion turns out at the appropriate time, inside a cylinder of radius $\lambda$, whose axis is the trajectory of considered particle. Of course, this model is meaningful only in the case when the average mean free path of particle $\lambda$, is much greater than its diameter: $\lambda \gg d$.

Since all particles encountered by selected particle are not fixed, as the average particle velocity should take its mean velocity, relative to other particles, and not relative to the vessel walls. The relative velocity is the difference between the velocities of two particles:

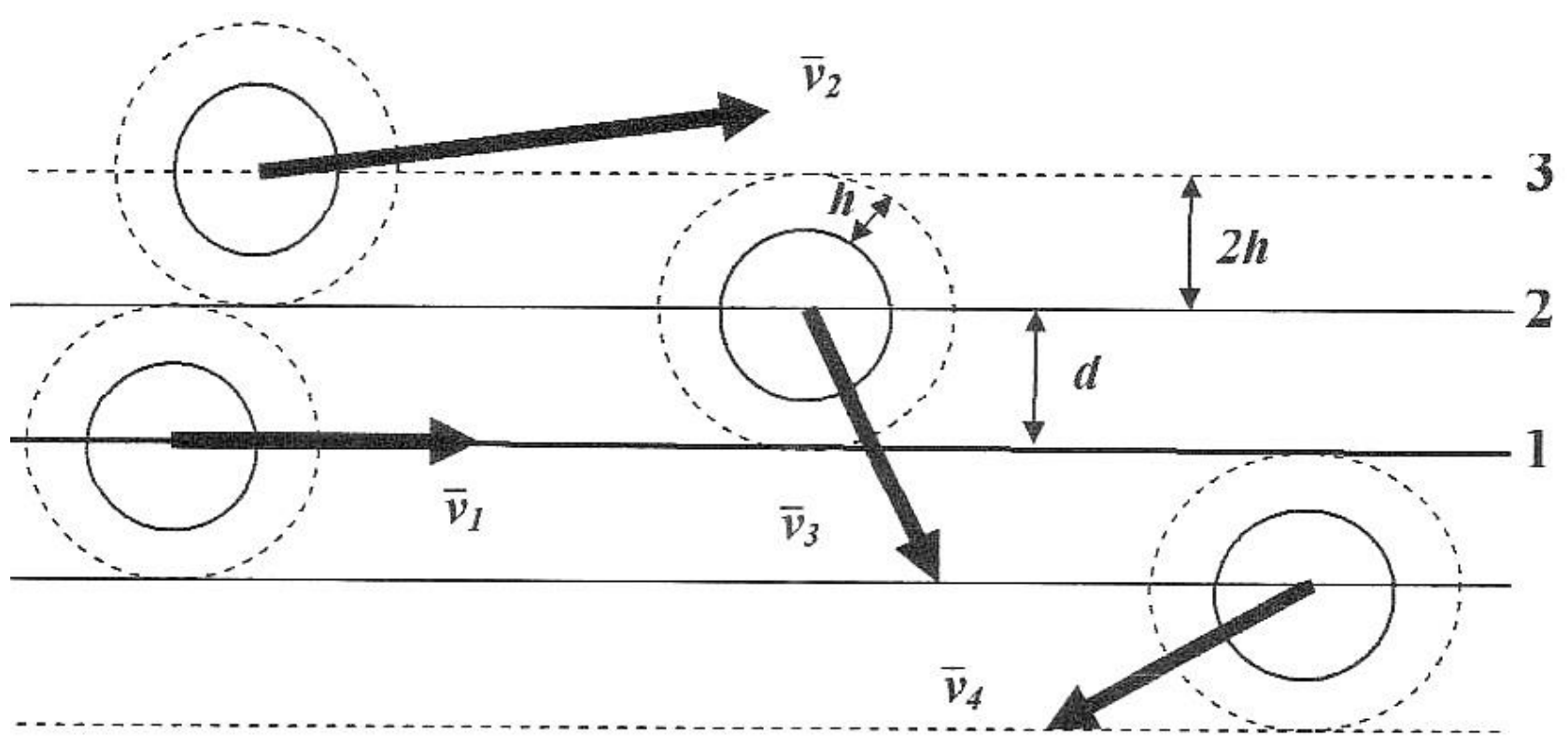

Figure 2. The collision of particles in sol.

1 - Motion trajectory of the selected particle. 2 - Border space volume where there are all the particles would have to have a collision with the selected particle. 3 - Boundary space volume where there are all the particles would have to have a collision with the selected particle if they are surrounded by an electrical double layer. $\boldsymbol{d}$ - Diameter of particles. $\boldsymbol{h}$ - Position of the maximum of the potential barrier created by the electric double layer around the sol particle.

$$
\overrightarrow{v_{\text {отн }}}=\overrightarrow{v_{1}}-\overrightarrow{v_{2}}
$$

We raise this equality in the square:

$$
\overrightarrow{v_{\mathrm{OTH}}^{2}}=\overrightarrow{v_{1}^{2}}-2 \overrightarrow{v_{1} v_{2}}+\overrightarrow{v_{2}^{2}}
$$

Since the average value of the sum is the sum of average values of the summands values, then we have

$$
\overrightarrow{v_{\text {отн ср }}^{2}}=\overrightarrow{v_{1 \mathrm{cp}}^{2}}-2 \overrightarrow{v_{1 \mathrm{cp}} v_{2 \mathrm{cp}}}+\overrightarrow{v_{2 \mathrm{cp}}^{2}}
$$


Since this approximation we assumed that the particle size distribution is unimodal character and the average velocity for all particles equally:

$$
\overrightarrow{v_{1 \mathrm{cp}}^{2}}=\overrightarrow{v_{2 \mathrm{cp}}^{2}}=\overrightarrow{v_{\mathrm{cp}}^{2}}
$$

Speed $\overrightarrow{v_{1}}$ and $\overrightarrow{v_{2}}$ two particles are statistically independent random variables, so we have:

$$
v_{R M S}^{2}=2 v_{a v^{2}}
$$

Therefore, as the average speed of the relative motion of the particles can take the mean square velocity of the particles:

$$
\left\langle v_{r}\right\rangle=\sqrt{2}\langle v\rangle
$$

During the time $\Delta \mathrm{t}$, selected particle will traverse the path

$$
l=\left\langle v_{r}\right\rangle \Delta t
$$

On this path, it collides with all particles were inside of this cylinder. The number of these particles is equal to the product of particles countable concentration $\boldsymbol{n}$ in the volume of the cylinder, ie, $n \pi d^{2} l$ or $n \sigma l$, where the value of

$$
\sigma=\pi d^{2}
$$

called the effective cross section of the particle. The average time between the last two selected particle collisions, with other particles, we find dividing the time $\Delta \mathrm{t}$ on the number of collisions:

$$
\tau=\frac{\Delta t}{n \sigma l}
$$

After transformations proceed to the formula

$$
\tau=\frac{1}{\sqrt{2} \sigma \iota v>n}
$$

Value $v$, inverse time $\tau$, represents the average number of collisions between a particle colloidal solution with other particles per unit time: 


$$
v=\frac{1}{\tau}=\sqrt{2} \sigma<v>n
$$

The mean free path particles $\lambda$, is the product of the average velocity of the mean time between collisions:

$$
\lambda=\langle v\rangle \tau
$$

hence we find that

$$
\lambda=\frac{1}{\sqrt{2} \sigma n}
$$

Considering expression for the effective cross section particles, obtain:

$$
\lambda=\frac{1}{\sqrt{2} \pi d^{2} n}
$$

We will estimate the average thermal velocity and the mean free path of the particles. $n=m / m_{\mathrm{Y}}$ countable concentration of colloidal particles $\left[1 / \mathrm{m}^{3}\right] ; m$ - mass concentration of the colloidal solution $\left[\mathrm{kg} / \mathrm{m}^{3}\right] ; m_{\mathrm{Y}}=\rho V_{\mathrm{Y}}$ - mass of colloidal particle $[\mathrm{kg}] ; \rho$ - the matter density of the colloidal particle $\left[\mathrm{kg} / \mathrm{m}^{3}\right] ; V_{\mathrm{u}}=\pi d^{3} / 6$ - volume of colloidal particle $\left[\mathrm{m}^{3}\right]$. Hence, the mass of the colloidal particle is:

$$
m_{\mathrm{Y}}=\frac{\rho \pi d^{3}}{6}
$$

Accordingly, the countable concentration will be:

$$
n=\frac{6 m}{\rho \pi d^{3}}
$$

Hence the final formula of the mean free path of the colloidal particle becomes:

$$
\lambda=\frac{\rho d}{6 \sqrt{2} m}
$$

This formula shows that for a fixed mass concentration of the colloidal solution the mean free path of the colloidal particle will grow in proportion to its diameter, as this decreases their partial concentration. In the case of fixed-size of particles their mean free path will decrease is 
inversely proportional to the mass concentration of the colloidal solution. The results of these calculations are presented in Figures 3 and 4.

The results show that at low concentrations of sols to $10 \mathrm{~g} / \mathrm{l}$, a sharp decrease of the mean free path of particles of the sol. Further increase of concentration leads to a more smooth decrease of this value.

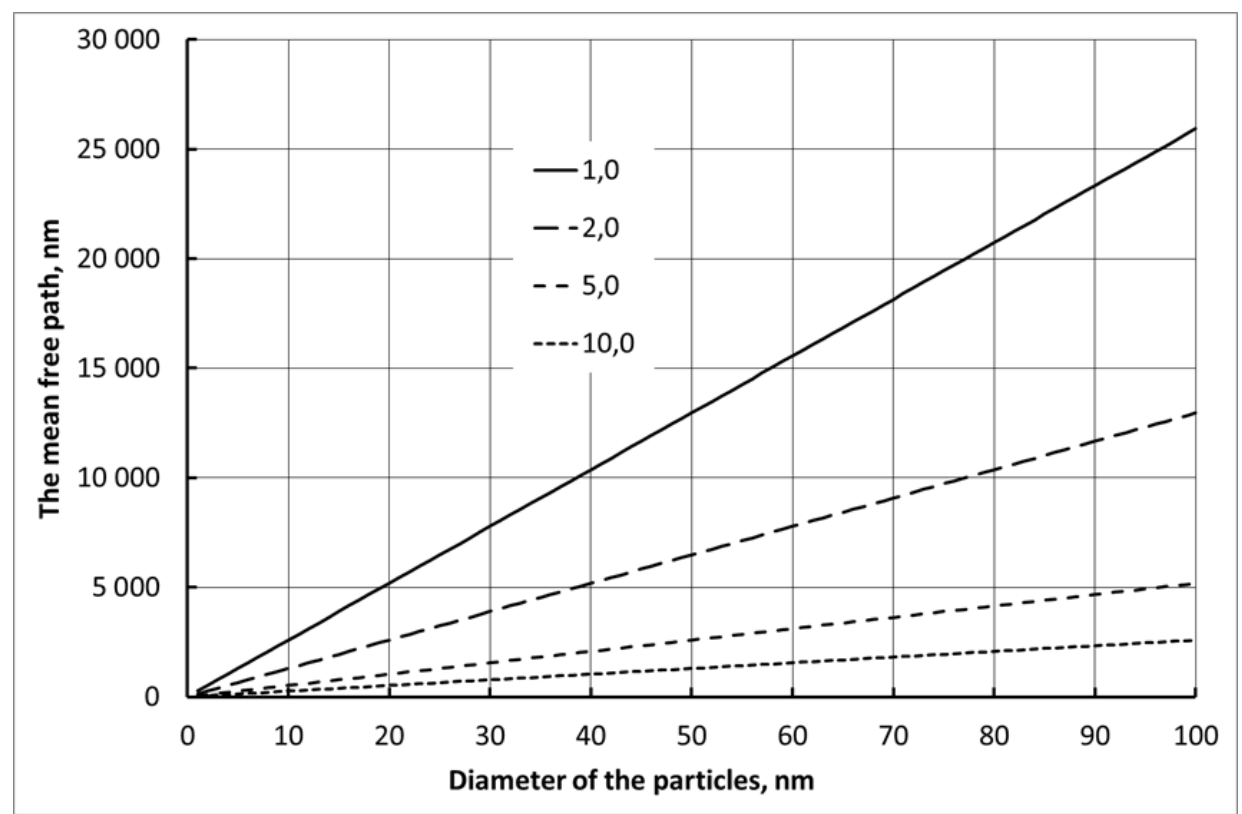

Figure 3. The results of the calculation of the mean free path of the particles in the silica sol depending on their diameter, for different mass concentration: $1.0 ; 2.0 ; 5.0 ; 10.0(\mathrm{~g} / \mathrm{l})$.

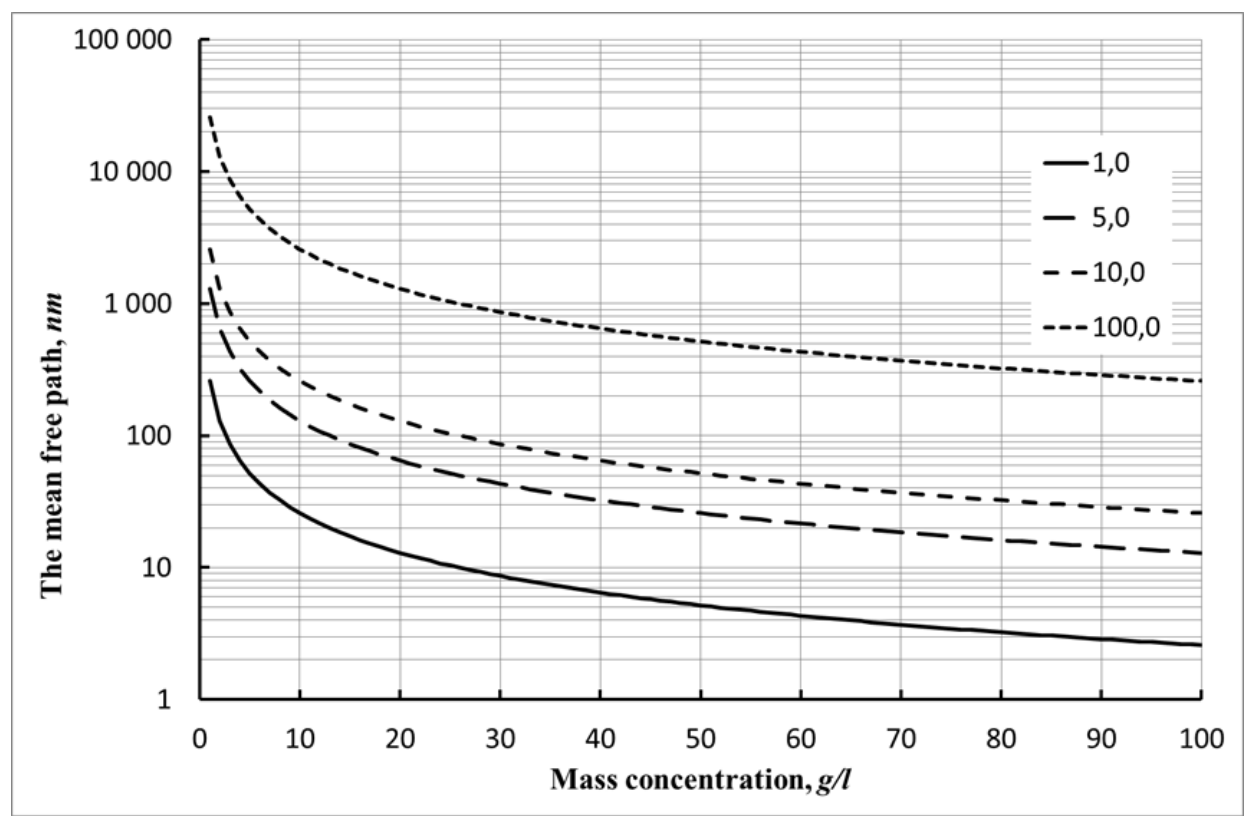

Figure 4. Results of the calculation of the mean free path of the particles in the silica sol depending on the mass concentration of particles of various diameters: $1.0 ; 5.0 ; 10.0 ; 100(\mathrm{~nm})$. 
Considering that the average speeds of the colloidal particles have the Maxwell distribution, the mean square velocity of the colloidal particles is:

$$
\langle v\rangle=\sqrt{\frac{3 k T}{m_{\mathrm{u}}}}
$$

Here, $k$ - Boltzmann constant, and $T$ - absolute temperature. Considering formula for the mass of colloidal particle, we obtain:

$$
\langle v\rangle=3 \sqrt{\frac{2 k T}{\rho \pi d^{3}}}
$$

The calculation results of average particle velocity for silica sol presented in Figures 5 and 6.

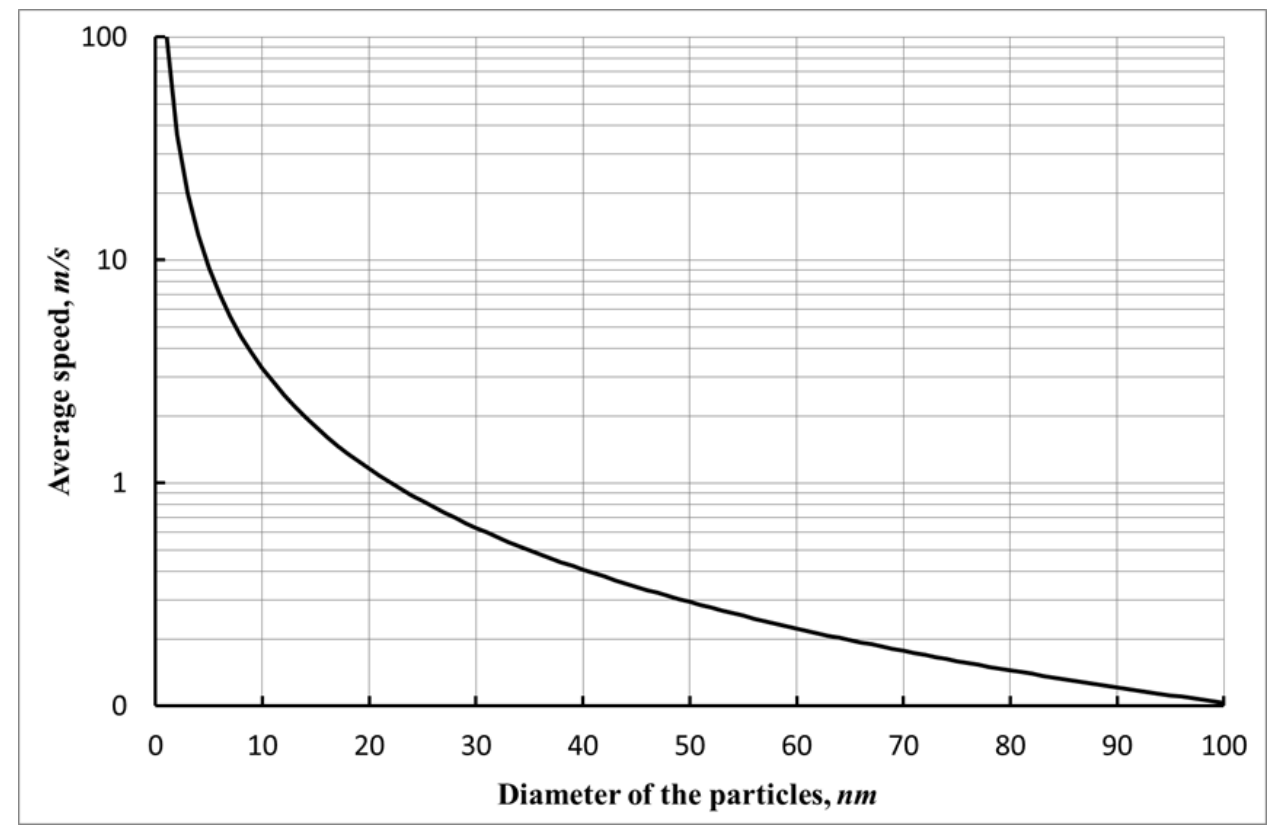

Figure 5. The calculation results of average particle velocity for silica sol. particles:

Now you can easily find the average collision rate of colloidal particle with other

$$
v=\frac{\langle v\rangle}{\lambda}=\frac{36 m}{\rho d} \sqrt{\frac{k T}{\rho \pi d^{3}}}
$$


The calculation results of the collision frequency of the silica sol particles depending on the particle size and the mass concentration of silica sol are shown in Figures 7 and 8.

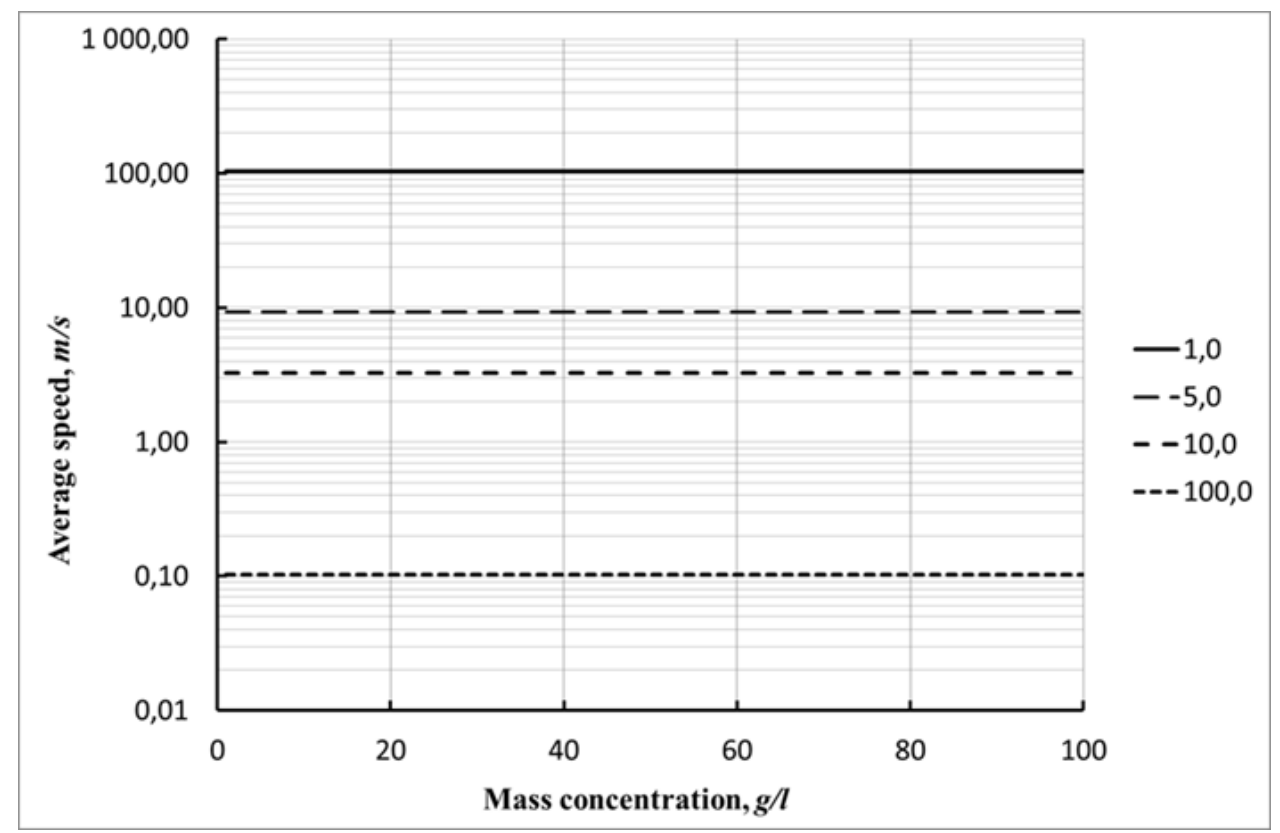

Figure 6. The calculation results of the mass concentration influence on the value of the average speed of the particles silica sol, for particles with different diameters: $1.0 ; 5.0 ; 10.0 ; 100(\mathrm{~nm})$.

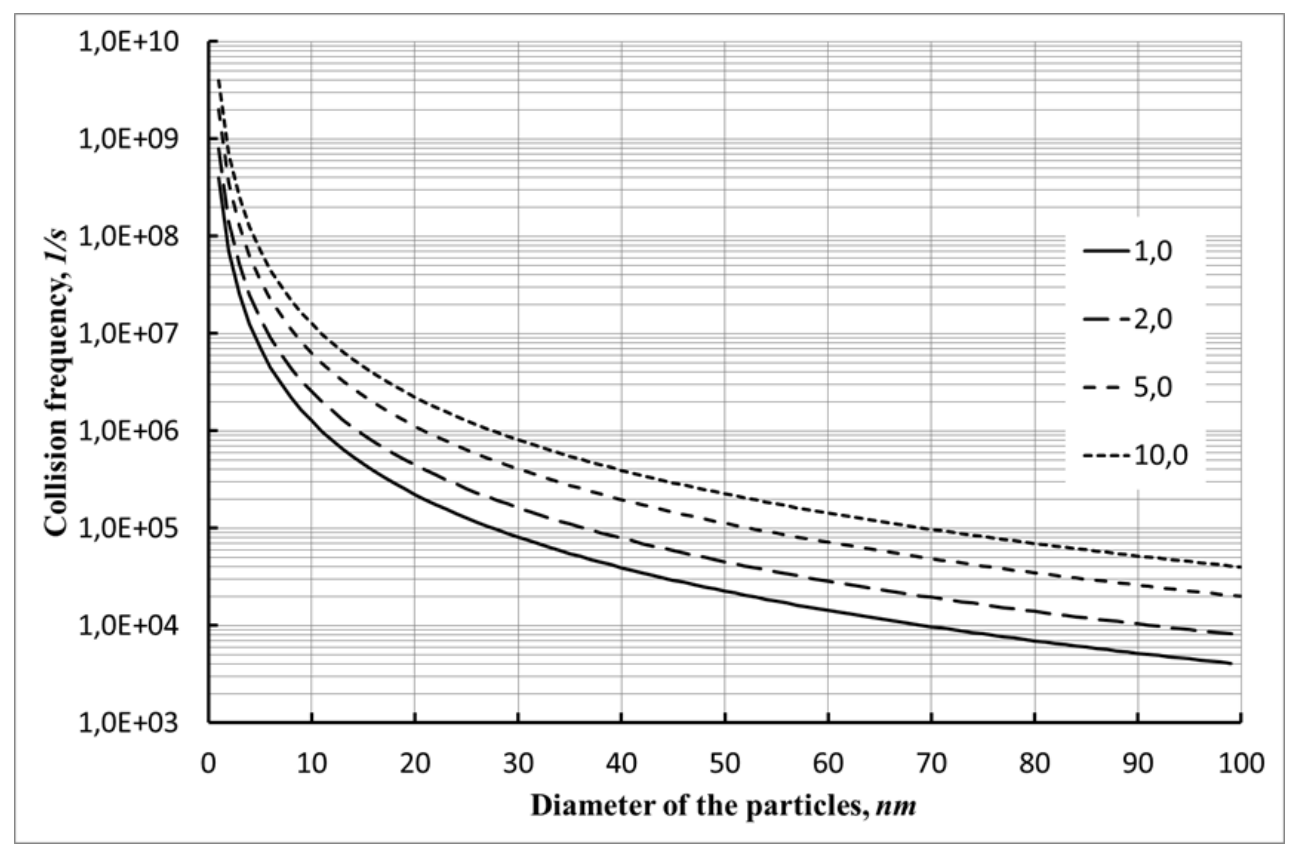

Figure 7. The calculation results of the collision frequency of the silica sol particles depending on their diameter, with different, mass concentration: $1.0 ; 2.0 ; 5.0 ; 10.0(\mathrm{~g} / \mathrm{l})$. 


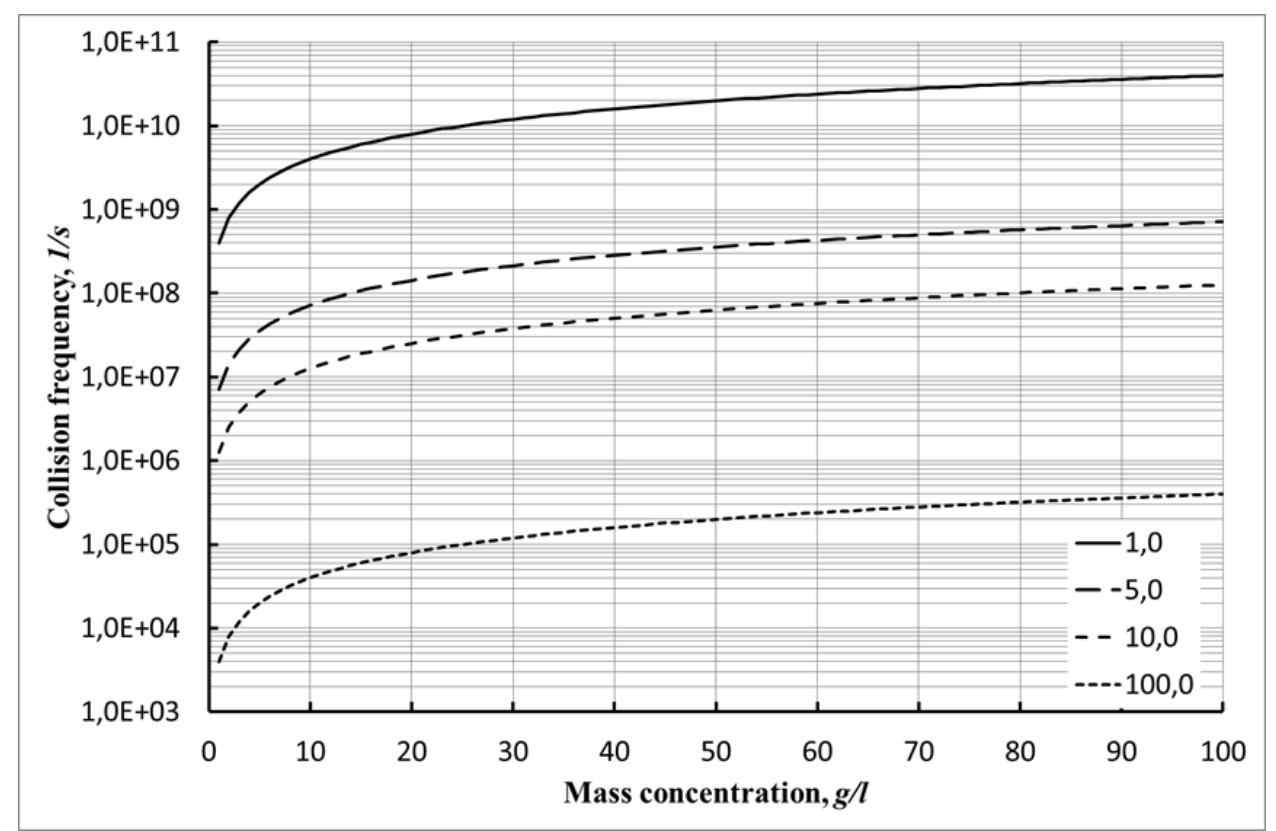

Figure 8. The The calculation results, the influence of mass concentration on the value of collision frequency of the silica sol particles, for particles with different diameters: $1.0 ; 5.0 ; 10.0 ; 100(\mathrm{~nm}) . \mathrm{T}=298.15 \mathrm{~K}$.

Consider a more complex model, where the particles are not elastic spheres, and have the electric double layer around them and are governed by the DLVO theory. In this case, the thickness of the electrical double layer will amount some value $\boldsymbol{h}$. This value corresponds to the position of the maximum of the potential barrier in the framework of the DLVO theory [11]. Hence, change the effective cross section particle - equation (4.92), and accordingly will be the value of:

$$
\sigma=\pi(d+2 h)^{2}
$$
into:

Hence the equation (4.61) (4.98) for the mean free path of the particle $\lambda$, is transformed

$$
\lambda=\frac{1}{\sqrt{2} \pi(d+2 h)^{2} n}=\frac{\rho d^{3}}{6 \sqrt{2}(d+2 h)^{2} m}
$$

Now, given the equation (4.106), we can easily find the average collision frequency of colloidal particle with other particles based on the DLVO theory. To simplify the conclusion of the corresponding equations, assume that an electric double layer does not change the mass of the particle as such: 


$$
v=\frac{36(d+2 h)^{2} m}{\rho d^{3}} \sqrt{\frac{k T}{\rho \pi d^{3}}}
$$

Hence, if we express the thickness of the electrical double layer, through a fraction of particle diameter:

$$
\alpha=\frac{h}{d}
$$

Obtain the relative change in the mean free path of the sol particles, depending on the relative thickness of the electric double layer:

$$
\frac{\lambda}{\lambda_{0}}=\frac{1}{(1-2 \alpha)^{2}}
$$

Where: $\lambda_{0}$ - free path length for the particles without an electric double layer according to formula (4.101).

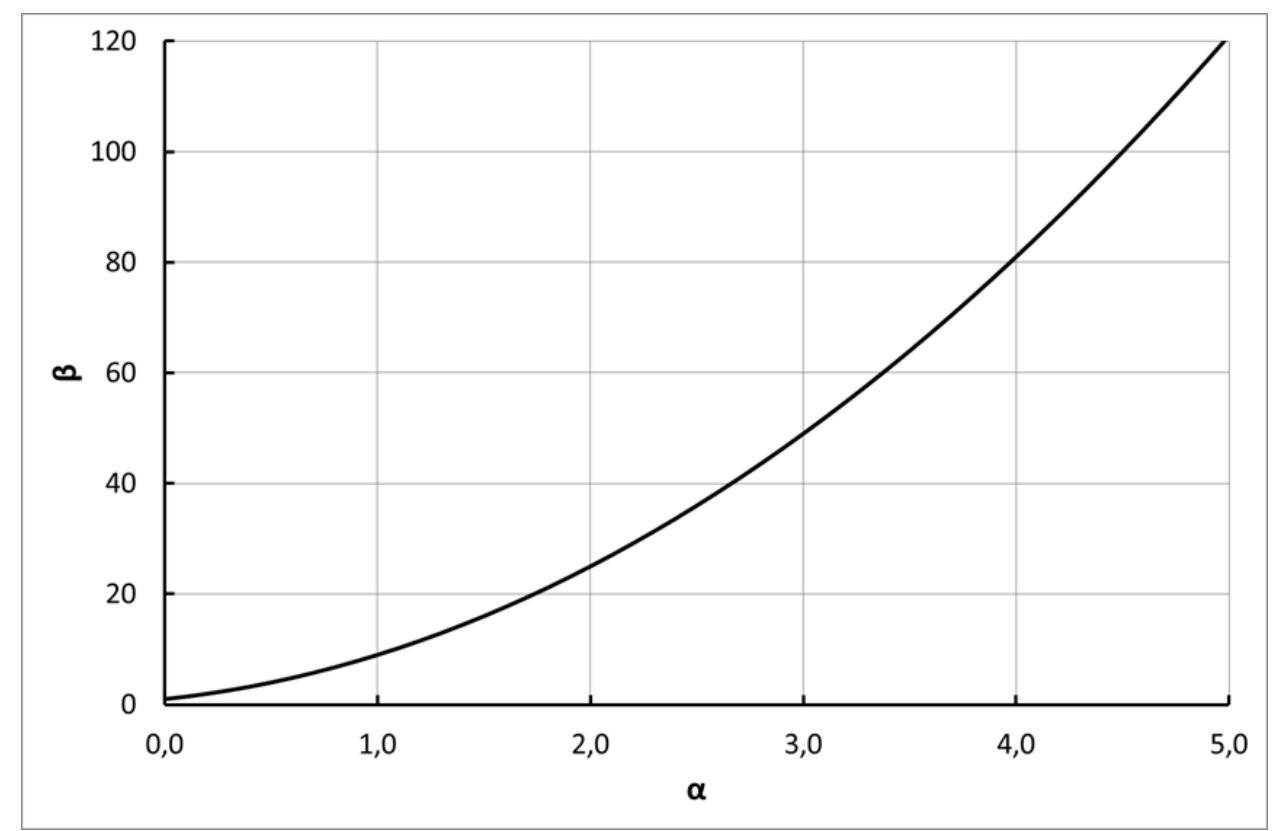

Figure 9. Dependence of the parameter $\beta$, which describes the relative changes in the frequency of collisions and the mean free path for the silica sol particles, depending on the relative thickness of the electrical double layer $(\alpha)$.

From this, also follows an equation for the relative change of frequency of collisions for particles of the sol, depending on the relative thickness of the electric double layer: 


$$
\frac{v}{v_{0}}=(1-2 \alpha)^{2}=\beta
$$

Where: $v_{0}$ - collision frequency for particles without electrical double layer in accordance with the formula (4.104). Accordingly, it turns out:

$$
\beta=\frac{v}{v_{0}}=\frac{\lambda_{0}}{\lambda}
$$

The obtained dependence is shown in Figure 9.

\section{CALCULATION OF THE NUMBER OF PARTICLES CAPABLE TO OVERCOME THE POTENTIAL BARRIER IN A COLLISION}

For overcome the potential barrier, the kinetic energy of the particle must be greater or equal to the potential barrier $E \geq E_{a k t}$. Accordingly, we obtain:

$$
E_{a k t}=\frac{m_{p} v_{0}^{2}}{2}=\frac{\rho \pi d^{3} v_{0}^{2}}{12}
$$

Where $v_{0}$ - minimum particle velocity at which it can overcome the potential barrier. From this we obtain:

$$
v_{0}=\sqrt{\frac{12 E_{a k t}}{\rho \pi d^{3}}}
$$

Maxwell distribution function for the particle speed is given by:

$$
F(v)=4 \pi\left(\frac{\alpha}{\pi}\right)^{\frac{3}{2}} v^{2} \exp \left(-\alpha v^{2}\right)
$$

Where: $\alpha=\frac{m_{p}}{2 k T}=\frac{\rho \pi d^{3}}{12 k T}$ - parameter Maxwell distribution function. Proceeding from equation (4.113), in accordance with (4.84), we obtain the fraction of particles from of their total number that can overcome the potential barrier of:

$$
\frac{C_{n}\left(v>v_{0}\right)}{C_{n 0}}=\int_{v_{0}}^{\infty} F(v) d v
$$


Substituting in (4.114) the function (4.113) and integrating, we obtain the following expression:

$$
\begin{aligned}
\frac{C_{n}\left(v>v_{0}\right)}{C_{n 0}} & =4 \pi\left(\frac{\alpha}{\pi}\right)^{\frac{3}{2}}\left(\frac{\sqrt{\pi}}{4 \alpha^{\frac{3}{2}}}+\frac{v_{0}}{2 \alpha} \exp \left(-\alpha v_{0}^{2}\right)-\frac{\sqrt{\pi}}{4 \alpha^{\frac{3}{2}}} \operatorname{erf}\left(\sqrt{\alpha} v_{0}\right)\right)= \\
= & 1+\frac{2}{\sqrt{\pi}}\left(\frac{E_{a k t}}{k T}\right)^{\frac{1}{2}} \exp \left(-\frac{E_{a k t}}{k T}\right)-\operatorname{erf}\left(\sqrt{\frac{E_{a k t}}{k T}}\right)
\end{aligned}
$$

Analysis of the resulting equations is shown in Figure 10.

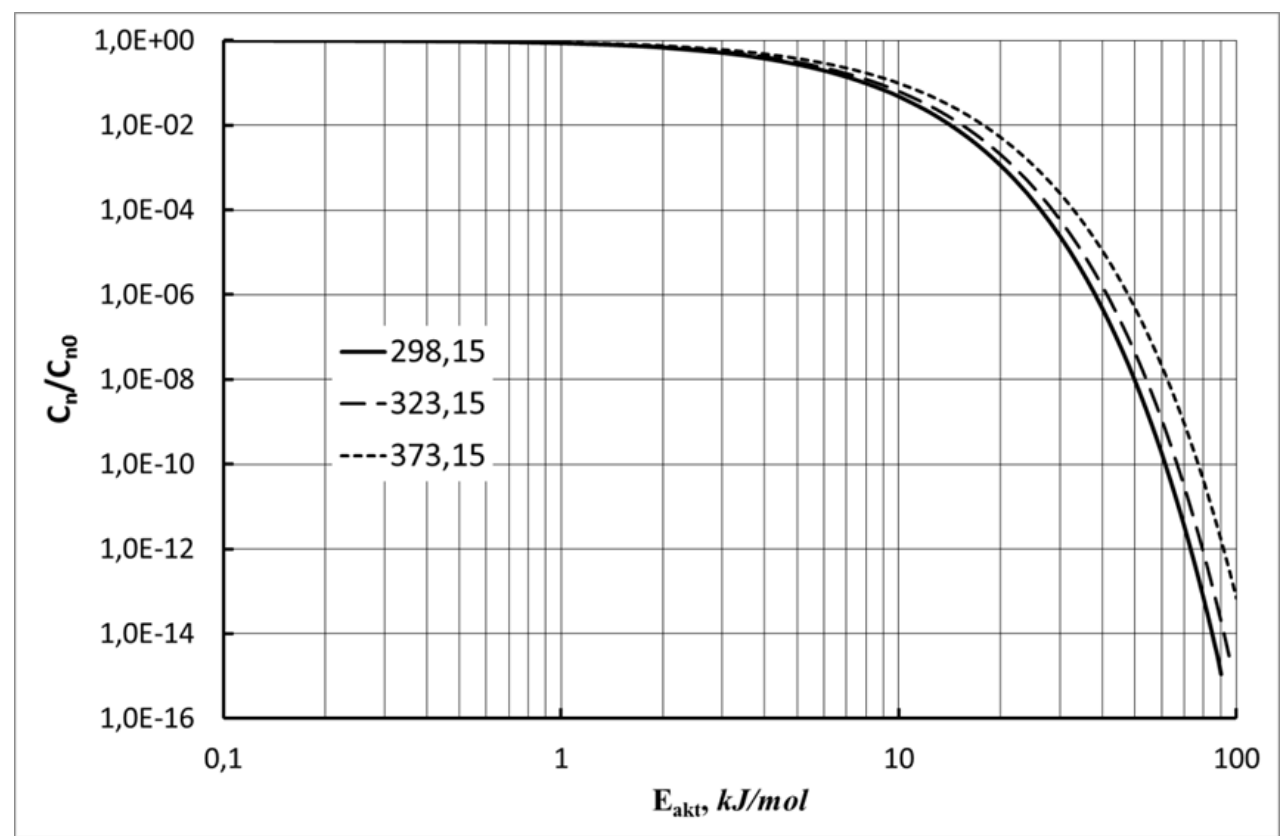

Figure 10. Dependence of the relative number of particles in the system those are able to overcome the potential barrier, depending on the value of this barrier. The calculation was made in accordance with equation (4.115) for systems are at a temperature of: $25^{\circ} \mathrm{C}, 50^{\circ} \mathrm{C}, 100{ }^{\circ} \mathrm{C}$.

From these data it is evident that the number of particles which are capable of overcoming the potential barrier is large for small values of this barrier. However, when the barrier height approximately above $10 \mathrm{~kJ} / \mathrm{mol}$, the ability of particles, to overcome this barrier, is significantly reduced. In addition, this effect is rather weakly dependent on temperature. Moreover, this dependence is stronger, the higher the value of this potential barrier (Figure 11).

For the understanding of physical meaning and nature of the influence on the final result of all three terms in the equation (4.115), we will hold a individual calculations of the values of these three members and assess their contribution to the total value of the relative number of particles in the system that are able to overcome a certain potential barrier. The obtained data are presented in Figure 12. 


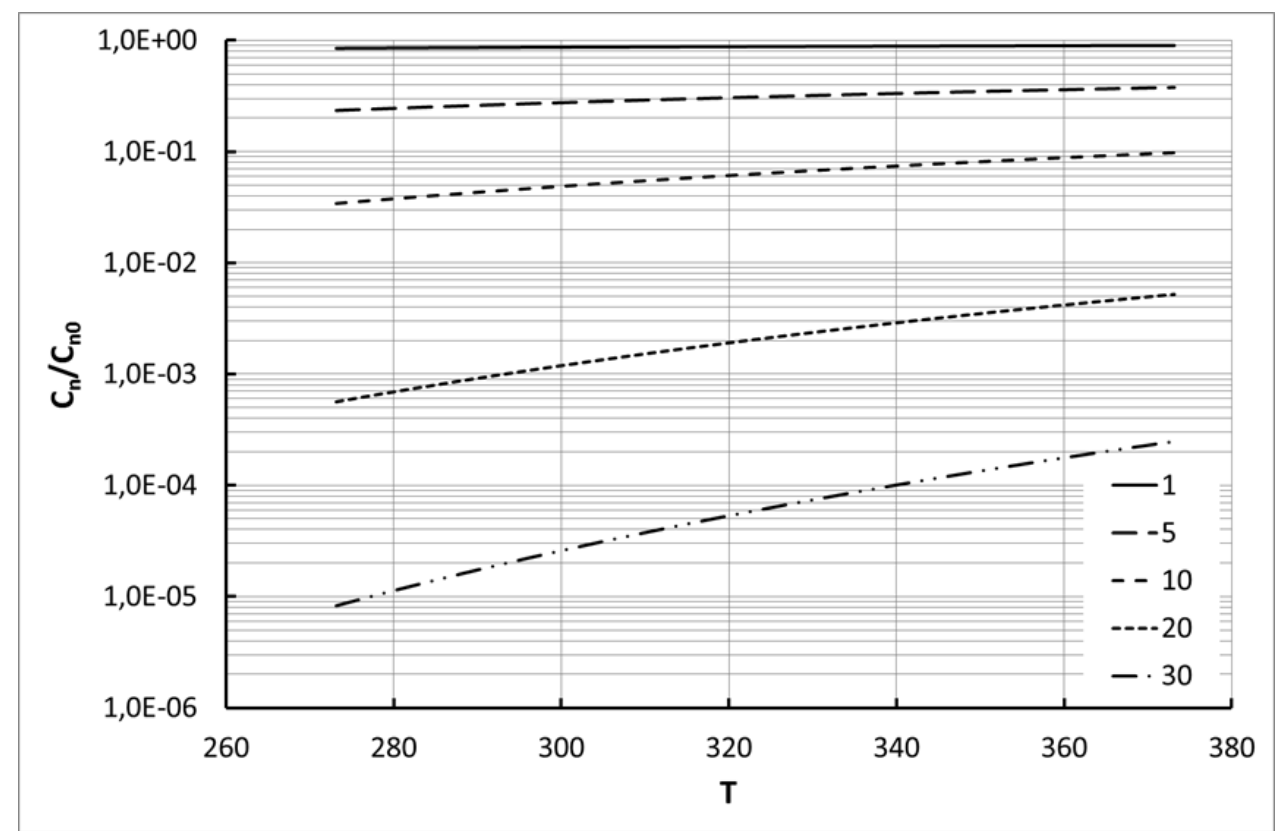

Figure 11. Dependence of the relative number of particles in the system is able to overcome the potential barrier, depending on the temperature. The calculation was made in accordance with equation (4.115) for systems having different values of the potential barrier $(\mathrm{kJ} / \mathrm{mol}): 1 ; 5 ; 10 ; 20 ; 30$.

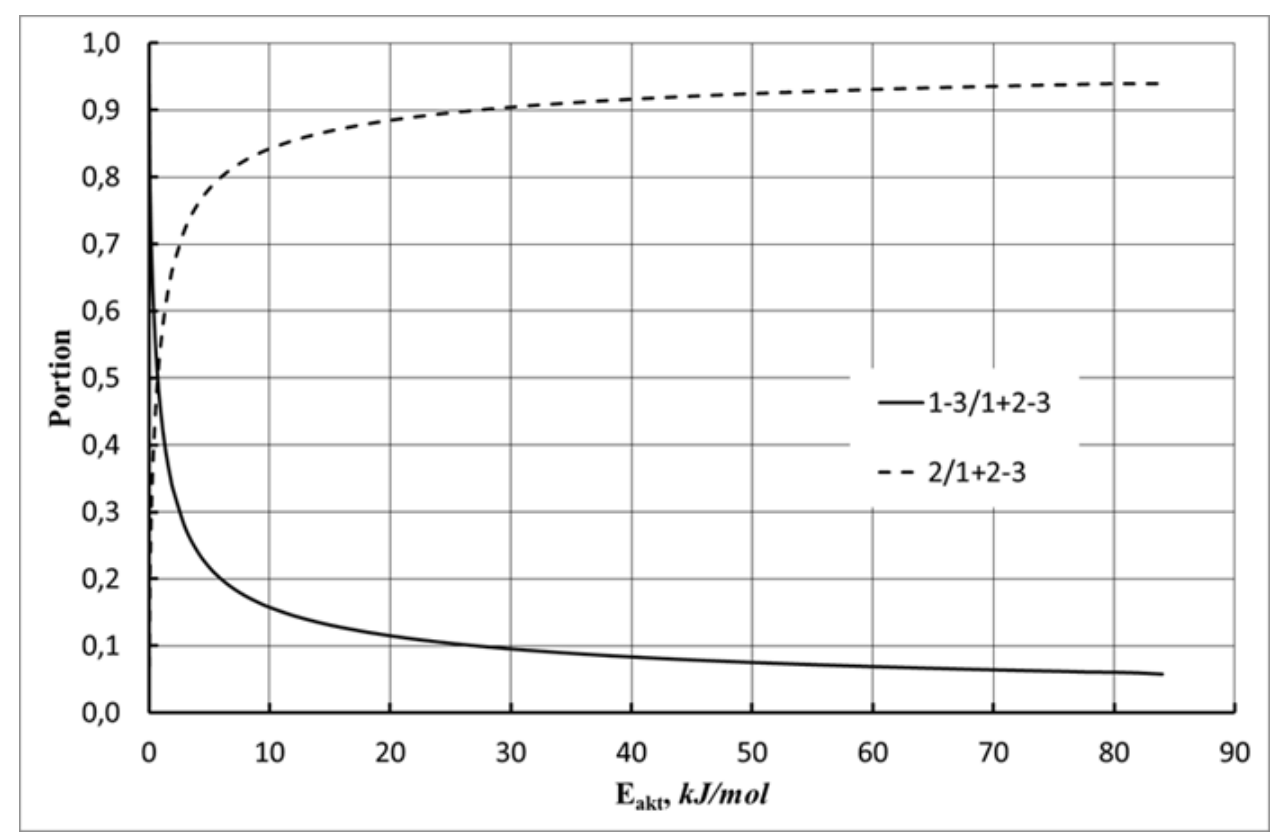

Figure 12. The relative proportions of the difference between the 1 and 3 terms in the equation (4.115) and the relative proportion of 2 term in equation (4.115).

The second term of this equation is the usual form, as in the Arrhenius equation. However, the physical meaning of the difference between the first and third terms of equation (4.79) is not obvious, although their contribution to the common component for the calculated value is high enough. 
Since this term includes, in its form, explicitly sets the error function, it can be assumed that this is an additional component of the particle speed dispersion. Further confirmation of this assumption is that the proportion of this component increases with decreasing value of the potential barrier. In addition, this equation indicates the possibility of a significant distortion of the results, in the calculation of the activation energy from experimental data in studies of processes of particle aggregation.

For such systems, in the case of conventional methods of linearization of the experimental data do not exclude the possibility of sufficiently high errors.

This possibility is related primarily to the presence of two variable functional members in equation (4.115). T

he calculation results of possible errors in the determination of the activation energy by a linear approximation of the logarithm of the relative number of particles in the system which are able to overcome the potential barrier, depending on the inverse temperature, by using the least squares method, shown in Figure 13.

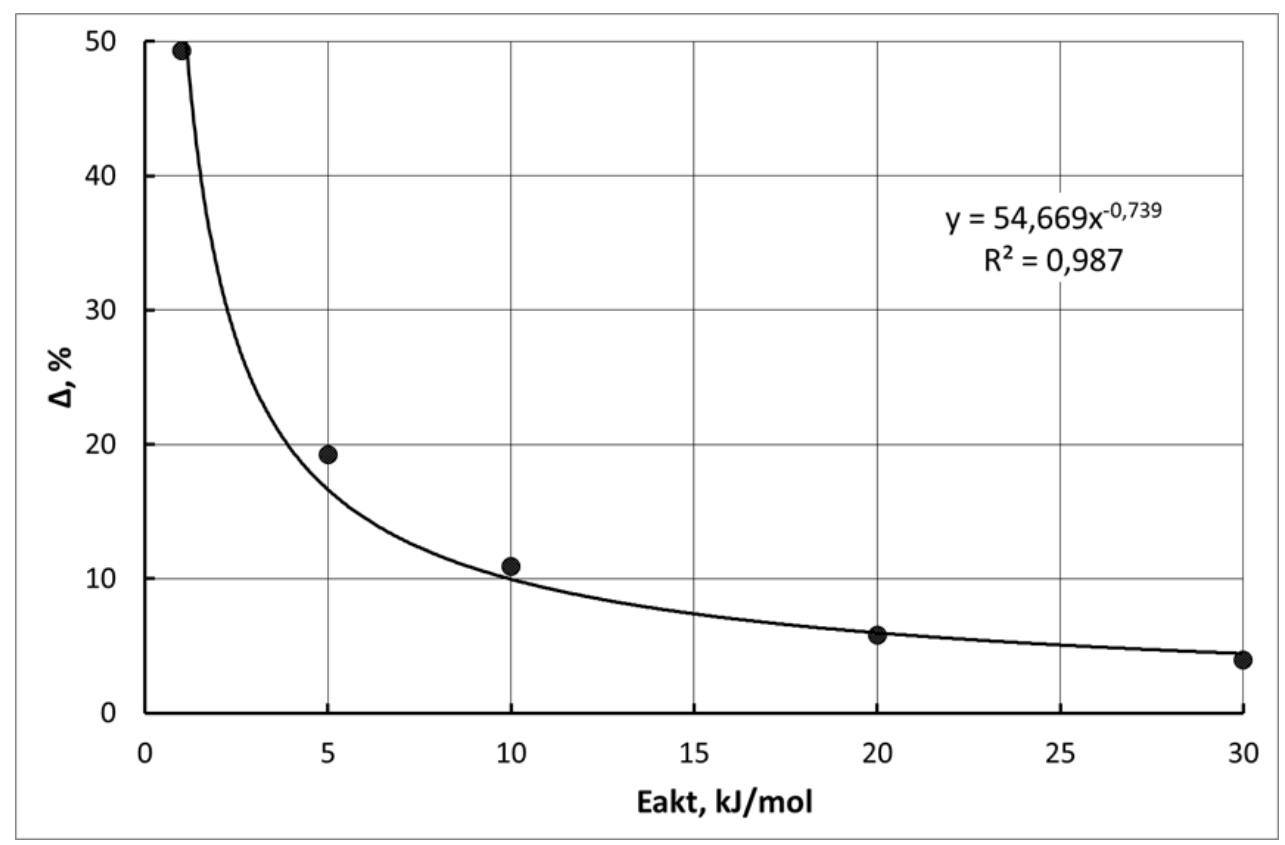

Figure 13. Calculation of the relative error, when determining the activation energy, for the aggregation processes of sols particles, depending on the value of activation energy in accordance with equation

(4.115).

From the data shown in Figure 13, it is seen that the character of the equation (4.115) leads to significant errors in the determination of the activation energy using conventional methods of linearization of the experimental data. These errors are particularly large for small values of the activation energy.

However, this equation allows us to precisely predict the kinetic characteristics of sols particle aggregation. This behavior is especially true for those cases when the shape and amplitude of the potential interactions between the sol particles are known with sufficient accuracy. 


\section{BASIC APPROACHES TO MODELING OF THE SOL-GEL TRANSITION KINETICS}

The sol-gel transition is a process of association of the sol particles, accompanied by the formation of an infinite aggregate of particles - aggregate that is commensurate with the size of the whole system. Nonlinear rate equations describing the processes of growth and destruction of aggregates are analogues of the nonlinear Boltzmann equation. Growth equation chains in discrete form was first obtained by M.V. Smoluchowski [12], and in continuous form first obtained by T. Schumann [13].

The essential difference between the Boltzmann equation and the equation of growth and fragmentation of aggregates lies in the fact that they are obey to various conservation laws. In the equation, of aggregates growth, their total mass is constant, but their total number in the growth process is not conserved, and is a variable quantity. The difference in the conservation laws largely determines the difference in the behavior of the solutions. For example, some solutions are suitable for describing the kinetics of gelation. These solutions correspond to the following conditions. First condition corresponds to the passage of the first stage of the process from the beginning until a certain point in time, which is the point of gelation. At this stage, for the sol particles and aggregates of finite size, is characterized by the following pattern, which consists in the fact that their total mass remains invariable. In the second step of the process, after the time of gelation, it begins to decrease. At this stage, there is a situation when in the system is formed, the mass flow from the finite size of aggregates, to the infinite aggregate, the so-called gel.

Consider the equation that describes the process of aggregate formation. Suppose that $\boldsymbol{c}(\boldsymbol{x}, \boldsymbol{t}) \boldsymbol{d} \boldsymbol{x}$ - average number of aggregates per unit volume at time $\mathrm{t}$, with the mass of the interval $(\boldsymbol{x}, \boldsymbol{x}+\boldsymbol{d} \boldsymbol{x})$. Units of mass $\boldsymbol{x}$ can be "born" as a result of a binary the coalescence of $(\boldsymbol{x}-\boldsymbol{y})$ aggregate and $\boldsymbol{y}$ - aggregate and "die" if they participate in the formation of larger aggregates. The rate of growth of aggregates is defined using a symmetric kernel $K(\boldsymbol{x}-\boldsymbol{y}, \boldsymbol{y})$. Rate of aggregates fragmentation denoted by $\boldsymbol{F}(\boldsymbol{x}, \boldsymbol{y})$. As a result, the equation of the reaction rate of growth and of fragmentation of aggregates takes the form:

$$
\begin{aligned}
\partial_{t}(x)=\frac{1}{2} \int_{0}^{x} K(x-y, y) c(x-y) c(y) d y & \\
& \quad-c(x) \int_{0}^{\infty} K(x, y) c(y) d y+\int_{0}^{\infty} F(x, y-x) c(y) d y-\frac{1}{2} \int_{0}^{\infty} F(y, x-y) c(y) d y
\end{aligned}
$$

If the variable describing the mass or dimensions of the unit takes discrete values, the equation of the reaction rate, the formation of $\boldsymbol{k}$ aggregates per unit volume is written as follows:

$$
\frac{d c_{k}}{d t}=\frac{1}{2} \sum_{j=1}^{k-j} \sum_{i=1}^{k-j} K_{i j} c_{i} c_{j}-c_{k} \sum_{j=1}^{\infty} K_{k j} c_{j}+\sum_{j=1}^{\infty} F_{k j} c_{j+k}-\frac{1}{2} c_{k} \sum_{j=1}^{k-j} \sum_{i=1}^{k-j} F_{i j}
$$


The greatest interest and convenience for the analysis of the solutions of equations (4.116) and (4.117) represent the corresponding moments of the distribution functions:

$$
M_{g}(t)=\int_{0}^{\infty} x^{g} c(x, t) d x=\sum_{k=0}^{\infty} x^{g} c_{k}(t)
$$

Here: $M_{0}(t)$ - the total number of aggregates; $M_{1}(t)$ - total mass of aggregates; $M_{2}(t)$ dispersion of aggregates in size, this value is proportional to the aggregation degree.

All these values are referred to the unit of volume. For some models of the growth and fragmentation of aggregates with specially selected functions $\boldsymbol{F}$ and $\boldsymbol{K}$, we can obtain closed equations directly for the functions $M_{k}(t)$. These equations are called equations of macroscopic rates. Their effect, in the general case is the total mass conservation equation $M_{1}(t)$.

Described equations are also used for modeling the processes polycondensation polymerization [14], and in meteorology for the calculation of the formation of atmospheric precipitation [15].

The literature describes the following forms kernels:

$$
\begin{aligned}
& K_{1}=1 ; \quad K_{2}=i+j ; \quad K_{3}=i j ; \quad K_{4}=(i j)^{\omega} ; \quad K_{5}=(i+j)^{v}(i j)^{\omega} ; \quad K_{6}=i f(j)+j f(i) ; \\
& K_{7}=\frac{1}{4}\left(i^{\frac{1}{2}}+j^{\frac{1}{2}}\right)^{3} ; K_{8}=\left(i^{\frac{1}{3}}+j^{\frac{1}{3}}\right)\left|i^{\frac{1}{3}}-j^{\frac{1}{3}}\right| ; K_{9}=\left(i^{\frac{1}{3}}+j^{\frac{1}{3}}\right)\left(i^{-\frac{1}{3}}+j^{-\frac{1}{3}}\right)
\end{aligned}
$$

Kernel $\boldsymbol{K}_{\mathbf{1}}, \boldsymbol{K}_{\mathbf{2}}, \boldsymbol{K}_{\mathbf{3}}$ - basic models the theory of polymerization of Flory-Stockmayer [16]. So $\boldsymbol{K}_{3}$ describes the kinetics of the sol-gel transition. In its turn the kinetic equations with modified a rate $\boldsymbol{K}_{4}$ and $\boldsymbol{K}_{5}$ allow you to simulate the transition to gelation with non-classical critical index [17]. In these kernels: $\omega$ - is a "geometric" index characterizing the surface area of large aggregates, while it is related with the fractal dimension of aggregates, which are formed in the process of such aggregation. Kernels $\boldsymbol{K}_{6}, \boldsymbol{K}_{\mathbf{7}}, \boldsymbol{K}_{\boldsymbol{8}}, \boldsymbol{K}_{\boldsymbol{9}}$ are intended for calculations of the formation of precipitation in the atmosphere. $\boldsymbol{K}_{\boldsymbol{6}}$ - allows you to obtain the general form of the equation for linear models [18]. $\boldsymbol{K}_{7}$ - describes the gradient coagulation [19]. $\boldsymbol{K}_{8}-$ describes the gravitational coagulation $[15,19] . \boldsymbol{K}_{\boldsymbol{9}}$ - designed to simulate the Brownian coagulation in the diffusion mode [19].

We construct a model of the rate of aggregation in terms of statistical thermodynamics. For colloidal particles having a double-well potential and be with a certain energy barrier, we write the equation of the aggregation rate of particles:

$$
k=k_{0} \exp \left(\frac{\Delta S}{k_{B}}\right) \exp \left(-\frac{E_{a k t}}{k_{B} T}\right)
$$

Where: $-k_{0}=\frac{2 k_{b} T}{3 \eta}$ the frequency of collisions with Brownian coagulation; $\eta$ - the viscosity of the medium.

Calculate the value of entropy changes: 


$$
S=k_{B} \sum_{i=1}^{\infty} f_{i} \ln f_{i}
$$
to:

Probability of the existence of aggregates with the size $\boldsymbol{i}, \boldsymbol{j}$ and $\boldsymbol{i}+\boldsymbol{j}$ are equal respectively

$f_{1}=\frac{i}{i+j} ; f_{2}=\frac{j}{i+j} ; f_{3}=\frac{i+j}{i+j}$

According to the law of Hess the entropy change:

$$
\Delta S=S_{2}-S_{1}
$$

Substituting (4.121), (4.122) and (4.123) into (4.120), we obtain:

$$
k=\frac{2 k_{B} T}{3 \eta}(i+j)(i j)^{\frac{i}{i+j} j} j^{\frac{j-i}{j+i}} \exp \left(-\frac{E}{k_{B} T}\right)
$$

We set $i=m j$ and by entering the function $\boldsymbol{u}$, from the equation (4.88) (4.124), we obtain:

$$
=\frac{k}{k_{0} i^{2}} \exp \left(\frac{E}{k_{B} T}\right)=(1+m) \cdot m^{\frac{m}{m+1}}
$$

$\boldsymbol{u}$ parameter of this equation is proportional to the rate of interaction between aggregates, and parameter $\boldsymbol{m}$ characterizes the ratio of the sizes of aggregates. Dependence of the parameter $\boldsymbol{u}$ from $\boldsymbol{m}$ is shown in Figure 14. Analysis of equation (4.124), and Figure 14 shows that when the ratio of the sizes of the interacting aggregates close to each other, aggregation rate is weakly dependent on $\boldsymbol{m}$. In the case of aggregates having a large difference in the sizes from each other, there has been a sharp increase in the rate of aggregation, which is proportional to $\boldsymbol{m}^{2}$. Thus, at occurrence of aggregates in this system that have a significant difference in the sizes among themselves, there will be a sharp increase in the rate of aggregation. Thus there is a process where large aggregates collected by themselves the individual particles and small aggregates. Absence of symmetry in this function relative to the point $\boldsymbol{m}=\mathbf{1}$, is likely indicates the presence of the particle flow from small to large aggregates and the absence thereof in the reverse direction.

For determining the time of the formation of an infinite aggregate investigate the asymptotic properties of the functions of the size distribution of aggregates for the model with the kernel $\boldsymbol{K}_{3}$. For this purpose let us write system of equations for the moment $\boldsymbol{M}_{2}$, which follows from equations (4.116) and (4.118), and is reduced to an ordinary differential equation: 


$$
\frac{d M_{2}(t)}{d t}=2 M_{2}(t)^{2}
$$

In this model, at time $\boldsymbol{t}=\boldsymbol{t}_{\boldsymbol{c}}$ is formed superparticle - an infinite aggregate. Tc parameter value is determined from the condition that the moment $\boldsymbol{M}_{2}(t)$, which is the degree of aggregation of reacting particles, becomes infinite. From (4.125) we find:

$$
M_{2}(t)=\frac{M_{2}(0)}{1-2 M_{2}(0) \cdot t}
$$

Hence we have:

$$
t=\frac{1}{2 \cdot M_{2}(0)}
$$

Further consideration we will carry the model for monodispersed particles of unit size:

$$
\begin{gathered}
c(g)=\delta\left(g-g_{0}\right) \\
M_{2}=g_{0}^{2}
\end{gathered}
$$

If $g_{0}=1$, we have $t_{c}=1 / 2$. To go from the dimensionless time to the true, we introduce aggregation time - the time interval between the individual elementary acts of aggregation:

$$
\tau_{a g r}=\frac{1}{k N}
$$

where: $N$ - number of particles per unit volume.

Hence the real aggregation time when $\boldsymbol{i}=\boldsymbol{j}=\boldsymbol{1}$ is equal to:

$$
\tau_{c}=t_{c} \cdot \tau_{a g r}=\frac{3 \eta}{8 k_{B} T N} \exp \left(\frac{E_{a k t}}{k_{B} T}\right)
$$

Analysis of equation (4.95) (4.130) shows that the gelation time is directly proportional to the viscosity of the medium is inversely proportional to the temperature and concentration of particles. Increase of the potential barrier in the interaction of particles leads to an increase in gelation time. 


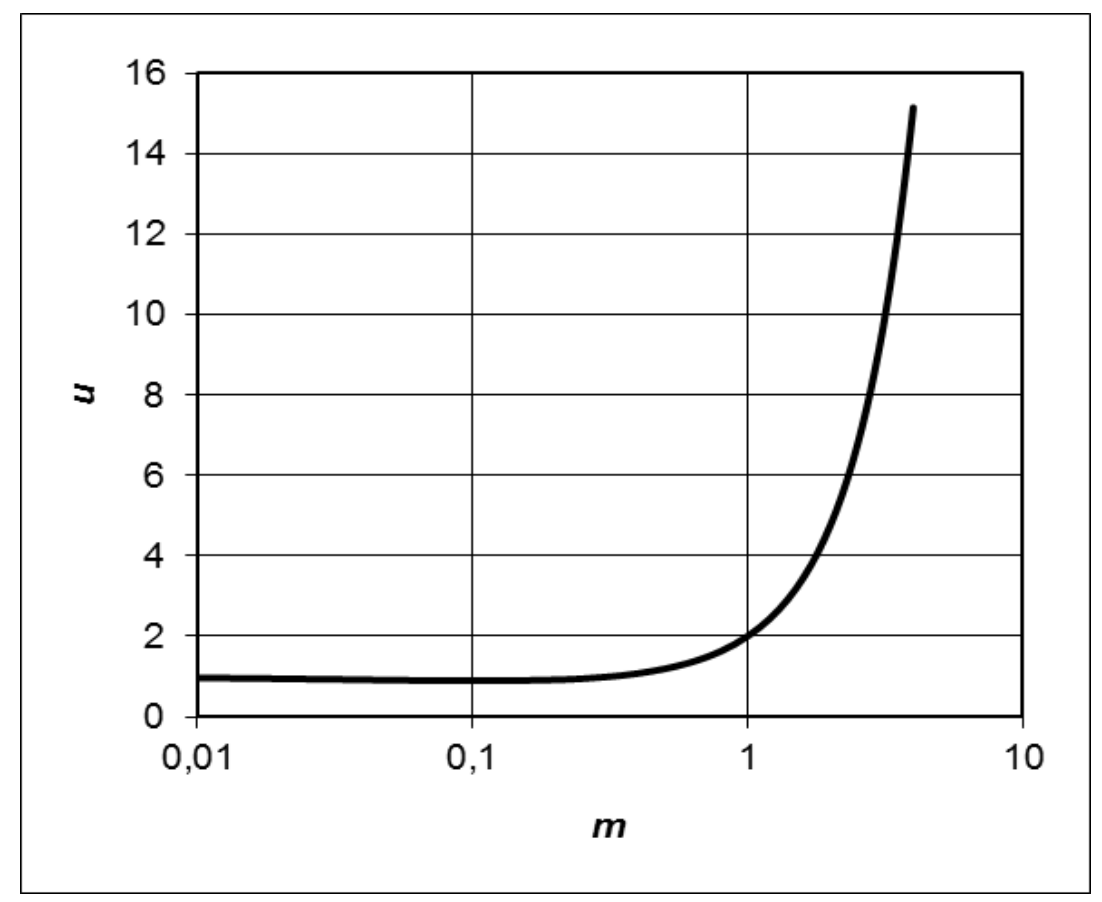

Figure 14. The relative rate of aggregates interaction among themselves dependence on the ratio of their sizes.

In order to determine the effect of hydrodynamic flows on the aggregation process, we consider the case when the unit is growing by consecutive attachment of individual particles to it. This process can occur due to the diffusive motion of the particles, and by the movement of aggregate under the action of hydrodynamic flows. In the latter case the speed of large aggregates is small compared with the thermal velocity of the particles, with the result that attachment of particles caused only by their diffusion. Given the both processes, the balance equation for the number of particles in the aggregate $\boldsymbol{n}$ has the form:

$$
\frac{d n}{d t}=\vartheta_{D}+\vartheta_{L}
$$

where: $\vartheta_{D}$ - frequency of sol particles sticking to the formed aggregate, with their diffusive motion; $\vartheta_{L}$ - frequency of particle sticking to this aggregate, due to their introduction into the traffic lane of the aggregate as it moves along a linear trajectory.

For $\vartheta_{D}$, assuming that size of the aggregate is large enough $(R \gg r)$, we have:

$$
\vartheta_{D}=4 \pi D R N
$$

here $D_{p}$ - diffusion coefficient of particles, $N$ - particle concentration.

In a second case:

$$
\vartheta_{L}=\pi R^{2} V N
$$


where $\boldsymbol{V}$ - speed of the aggregate under the action of hydrodynamic flow.

Aggregate, which is formed at the sol-gel transition has fractal geometry, hence the number of particles in it, respectively, equal to:

$$
n=\left(\frac{R}{r_{0}}\right)^{D_{f}}
$$

Where: $D_{f}$ - fractal dimension.

Substituting equations (4.132)-(4.134) into equation (4.131) we obtain:

$$
\frac{d n}{d t}=\vartheta_{0} \cdot n^{\frac{1}{D_{f}}}+\vartheta_{1} n^{\frac{2}{D_{f}}}
$$

Where: $\vartheta_{0}=4 \pi D r_{0} N, \vartheta_{1}=\pi r_{0}^{2} V N$.

Determine the time in which all the sol particles gather in one aggregate, which is equal to the size of the whole system:

$$
\tau=\int_{0}^{\infty} \frac{d n}{\vartheta_{0} \cdot n^{\frac{1}{D_{f}}}+\vartheta_{1} n^{\frac{2}{D_{f}}}}=\frac{\pi \cdot \vartheta_{0}^{D_{f}-2}}{\vartheta_{1}^{D_{f}-1} \sin \left(D_{f}-1\right) \pi}
$$

Compared to (4.130), equation (4.136) gives more overestimated results in gelation time, since it does not account the coalescence of aggregates. However, an analysis of the asymptotic behavior of equation (4.136) shows that it gives results similar to the equation (4.130). Thus, from equation (4.136) implies that the gelation time is inversely proportional to the particle concentration:

$$
\tau=\frac{2^{2 D_{f}-3} D^{D_{f}-2}}{d^{D} V^{D-1} N \sin \left(D_{f}-1\right) \pi}=\frac{B}{d^{D_{f}} V^{D_{f}^{-1}} N}
$$

Where: $d=2 r_{0}-$ is the particle diameter.

So from (4.137) that the gelation time is inversely proportional to countable concentration of particles:

$$
\tau \sim \frac{1}{N}
$$

Accordingly, speed of movement of aggregates has the following proportion from the gelation time: 


$$
\tau \sim \frac{1}{V^{D_{f}-1}}
$$

To determine the nature of the proportionality of the particle size, it is necessary to determine the nature of the influence of other factors on this relationship, such as the diffusion coefficient D. The diffusion coefficient of the particles depends on the average speed of movement of particles and their mean free path [20] and using equations (4.101) and (4.103) will have the form:

$$
D=\frac{1}{3}\langle v\rangle \lambda=\frac{1}{6 m \sqrt{\pi}} \sqrt{\frac{k T \rho}{d}} \sim d^{-\frac{1}{2}}
$$

In view of the above factors for the size the precursor particles we have the following proportionality if the concentration of the sol is constant and is expressed in counting units:

$$
\tau \sim d^{1-\frac{3}{2} D_{f}}
$$

If the experiment is kept constant mass concentration, this formula will change the appearance and will look as follows:

$$
\tau \sim d^{4-\frac{3}{2} D_{f}}
$$

The shape of the aggregate and its fractal dimension $D_{f}$ may be different, depending on the nature of aggregation, and takes values from literature data from the 1.75 to $2.5 \div 3.0$ $[21,22]$.

To assess this relationship experiments were performed to study the effect of particle size of the sol, on its gelation time. In all experiments, was maintained constant mass concentration of the sol. Obtained dependences are presented in Figure 15.

Figure 15 shows that the obtained dependences are well fit into a linear correlation. In this case, using the relation (4.141), we can calculate the fractal dimension of the resulting gels. So for a temperature of $80{ }^{\circ} \mathrm{C}$, it is $D_{f}=1.4407 \pm 0.0023$, and for a temperature of $100{ }^{\circ} \mathrm{C}$ $D_{f}=1.4100 \pm 0.0095$.

Such small values of fractal dimension, of emerging gels indicate their very open structure. Significant cause of such differences in the value of the fractal dimension as compared with literature data can only be explained by the fact that in the published literature were investigated gels which have been dried and at them have already undergone the process of syneresis, i.e. sealing.

In our case, the proposed method we have studied the formation of the original gel containing all the water that was in the starting part of the sol. The remaining particles are included in the structure after the gelation point. Thus, the fractal dimension of the infinite aggregate should increase upon completion of its formation after passing through gelation point. 


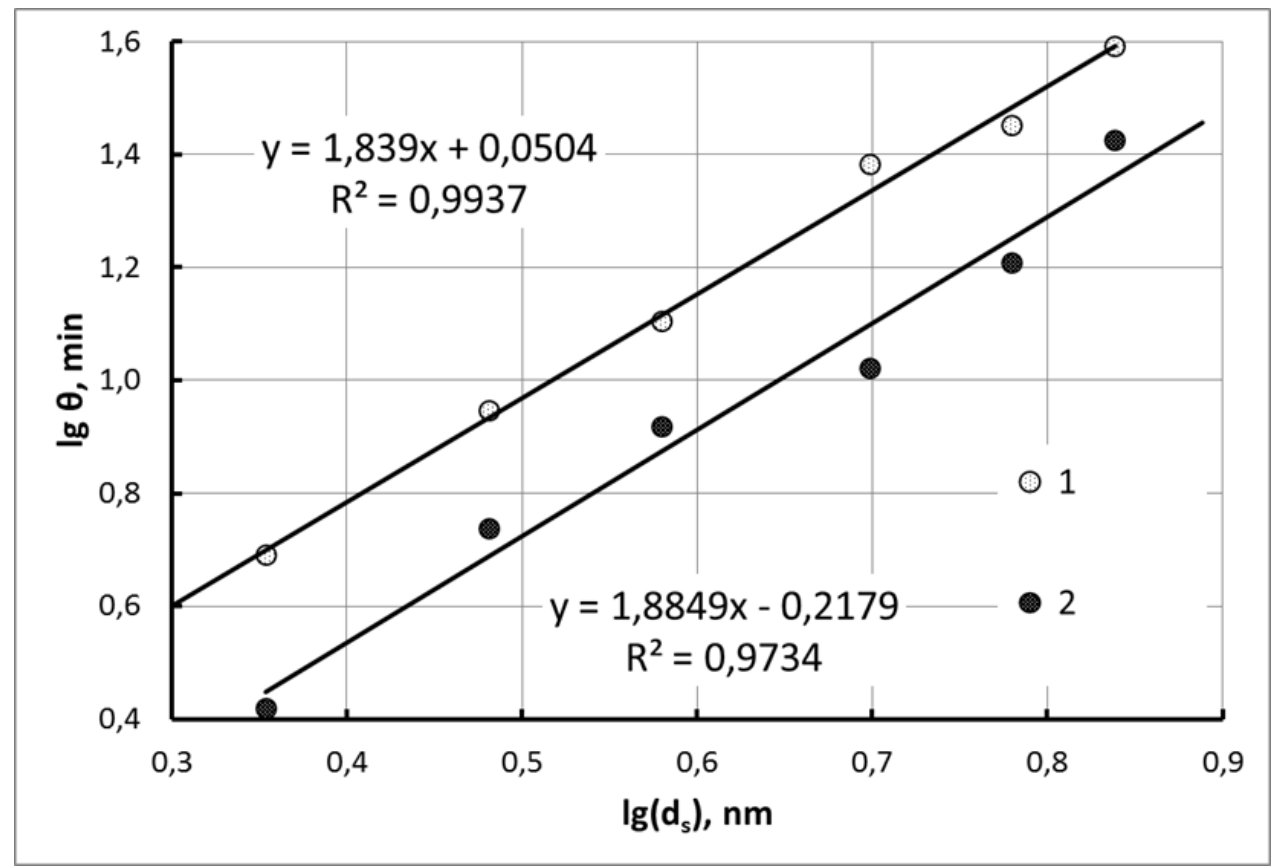

Figure 15. Dependence of gelation time, on the size of the colloidal particles in the sol at various temperatures. Axis of the figure plotted in logarithmic coordinates. $1-80^{\circ} \mathrm{C} ; 2-100{ }^{\circ} \mathrm{C}$.

In addition, from the obtained data it is seen that increasing the temperature of the process leads to the formation of a more open structure of the gel. These is apparently due to the increase in the rate of gel formation, and respectively reduce the possibility of penetration of the sol particles into the interior of aggregate and reduce the average coordination number of the sol particles in the aggregate with their interaction with each other.

Of course, should not be discounted, the moment that suggested above the theoretical premises are based on a number of assumptions and approximations. Therefore, for this reason also the possibility of certain errors in the evaluation of certain parameters in complex systems such as silica sols. Therefore, further research in this area should be directed at finding additional factors have an impact on the character of the sol-gel processes, as well as the nature of the structuring of emerging gels.

\section{FORMAL KINETIC DESCRIPTION OF THE SOL-GEL PROCESS}

It must be recognized that in order to describe the system in the process of gelation, it is necessary to know the way that it goes in the conversion of the starting sols into the products gels, nanocomposite materials. Such knowledge would allow conscious control sol-gel transformation. In other words, we must know the mechanism of this transformation. It is also important and the time evolution of the transition of the reaction system from the initial state (initial sols or precursors) to the final state - products sol-gel synthesis. This indicates the fact that is necessary information about how quickly occurs, the process of their formation. For answers to these questions can be used independent field of chemical knowledge, studies the reaction mechanisms and patterns of their occurrence in time - chemical kinetics. 
The fundamental concept of chemical kinetics is the concept of reaction rate. The reaction rate is defined as the change in the amount of reactant per unit time, and is related to one of the reaction space.

Strictly speaking, the colloidal system is inherently heterogeneous system. However, because the size of the colloidal particles is very small compared to the size of the entire system, therefore, with certain assumptions, for the application to these systems may be used to describe the laws of molecular systems. Such a system can be regarded as quasi-homogeneous.

This approach has long been used in physics. So there is the term "quasiparticle" - a concept in quantum mechanics, the introduction of which can significantly simplify the description of complex quantum systems with internal interaction, such as solids and quantum liquids.

For example, an extremely complex description of the motion of electrons in semiconductors can be simplified by introducing a quasiparticle called electron conductivity different from the electron mass, and moving in free space. For the description of vibrations of the atoms in the lattice sites in the theory of condensed matter, use phonons to describe the propagation of elementary magnetic excitations in the system of interacting spins - Magnons.

The idea of using the quasiparticles was first proposed by Landau, to Fermi-liquid theory, to describe the liquid helium-3, and later it was used in the theory of condensed matter. To describe status of such systems directly solving the Schrödinger equation for $10^{23}$ interacting particles, it is impossible. Circumvent this difficulty can be by reducing the problem of interaction of a plurality of particles to a simple problem with a non-interacting quasiparticles [23]. Similarly, we can consider other systems, in particular - colloidal systems.

As an example of quasi-homogeneous approach to the description of heterogeneous systems, we can give an example of the description of processes in porous media. In accordance with the quasi-homogeneous model, the disperse medium is represented as a continuous [24]. Thus, the most traditional method of describing transport processes in porous media is a quasi-homogeneous approximation, based on replacing the real dispersion medium by a continuous medium with effective parameters. For this purpose we introduce the effective parameters - coefficients of heat transfer, and mass transfer in volume of quasi-homogeneous porous body, the effective reaction rate constants. In this case, the heterogeneous reaction, formally regarded as homogeneous. To account for the influence of the geometry of the phase interface often used corresponding simplified geometric models for the structure of the medium. However, the quasi-homogeneous approximation in some cases is not sufficiently precise [25].

Depending on whether the process is quasi-homogeneous or heterogeneous, the reaction space concept is different. In the quasi-homogeneous system process is performed in the entire volume of the system, in a heterogeneous system, - at the interface. An example of such a system is the deposition of the sol particles on a solid substrate. Mathematically, this is written as:

quasi-homogeneous process:

$$
r= \pm \frac{d n}{V d t}
$$

heterogeneous process:

$$
r= \pm \frac{d n}{S d t}
$$


The sign in front of the derivative is formally reflects the situation taking place during the process, namely, what is happening with this substance, it is expended or accumulated. $\boldsymbol{n}$ Number of particles participating in this moment in this process, in this system. If in the process of quasi-homogeneous system volume remains constant (closed system), we have:

$$
\frac{d n}{V}=d C_{n}
$$

Therefore the rate associated with a change in the countable concentration $\left(C_{n}\right)$ of the reactant with time:

$$
r= \pm \frac{d C_{n}}{d t}
$$

Assumed that the rate of reaction - positive value. The mathematical basis for a quantitative description of the reaction is the basic postulate of chemical kinetics - the law of mass action. In the kinetic formulation of the law expresses proportional to the rate with concentrations of the reactants:

$$
r=k \prod_{i} C_{i}
$$

Where: $\boldsymbol{k}$ - rate constant of the reaction. This is an important kinetic parameter, formally reflecting the values of the reaction rate at the concentrations of the reactants are equal to one. The rate constant is independent of concentration and time, but for most of the reactions is temperature dependent. The index $i$ - refers to the $i$-th type of reacting particles.

In formal kinetics is believed that if the conversion of the starting reactants into products is not accompanied by any intermediate materials or particles, i.e. proceeds in one step, such a reaction is simple or elementary. This approach can also be applied to describe the kinetics of the interaction of the colloidal particles.

Consider the direct kinetic problem for the cases of passing in a simple reactions between the particles in a closed isothermal system (volume and temperature are constant). In this case, a simple reaction scheme, which goes in one step, and wherein only one reagent is involved, the particles of type A, can be written as:

$$
n A \stackrel{t}{\longrightarrow} \text { Substances }
$$

Where: $\boldsymbol{n}$ - the order of the reaction in this case are the same in magnitude, with particle numbers that participate in interaction of elementary act. Depending on the value of $\boldsymbol{n}$, can be distinguished cases of mono-, bi-, and three-particle interactions involving one type of the reacting particles. Mathematical model of such reactions can be represented by a differential equation:

$$
\frac{d C_{A}(t)}{d t}=-k C_{A}(t)^{n}
$$


With initial conditions corresponding to the concentration of the particles $\mathbf{A}$ at the start of the reaction $(\boldsymbol{t}=0): C_{A}(0)=C_{A 0}$.

Concentration $C_{A 0}$ called initial concentration and the value $C_{A}(t)$ at each time - the current concentration. Analytical solution of the direct kinetic problem is to establish a functional relationship of current concentration with time.

After a number of obvious transformations we obtain the general solution of the direct kinetic problem, which allows us to write the current dependence of the concentration of the reagent depending on time:

$$
k=\frac{1}{(n-1) t}\left(\frac{1}{C_{A}^{n-1}}-\frac{1}{C_{A 0}^{n-1}}\right)
$$

Obviously, depending on the order of the reaction, the concentration of the precursor particles decreases with time in various ways. For example, if for the order of the reaction is formally to attribute the values 0,2 , or 3 , and then we obtain the following expression. As we see, the equation in the form (4.109), is not applicable for a first order reaction because for $\boldsymbol{n}=$ 1 , it comprises uncertainty of the $0 / 0$ type. This uncertainty can be opened by L'Hospital's rule. Thus, in a first order reaction, the flowing reagent concentration decreases with time according to the exponential law:

$$
\begin{aligned}
& n=0 ; \quad C_{A}(t)=C_{A 0}-k t \\
& n=1 ; \quad C_{A}(t)=C_{A 0} \exp (-k t) \\
& n=2 ; \quad C_{A}(t)=\frac{C_{A 0}}{1+k C_{A 0} t} \\
& n=3 ; \quad C_{A}(t)=\frac{C_{A 0}}{\sqrt{1+2 k C_{A 0}^{2} t}}
\end{aligned}
$$

Obtained according to (4.145)-(4.148) is called the equation of the kinetic curves. In practical terms, the kinetic curves can be conveniently represented in the form of graphs. So in the figure 16 shows the form of the kinetic curves showing the consumption of reagent in hypothetical reactions having different orders, but equal, numerical values of the rate constants, and the initial concentration of the reacting substance. In fact, these equations analysis was conducted for their dimensionless form. This indicates that they are universal and can be used to solve different problems.

From the dimensional and similarity theory it is known that the numerical values of the variables and coefficients of the equations depend on the choice of scales, dimensions or characteristic values. Bad choice of dimensions, because of the limited number of bits to represent numbers in computers, can lead to loss of accuracy when performing arithmetic operations with very large and very small numbers. Therefore, it is important to have a good 
scaling of the variables, that is, to move from dimensional to dimensionless variables with a reasonable choice of scale dimensional variables. Selection of scale or, in other words, the choice of the characteristic values of the physical quantities made so that the dimensionless variables did not differ greatly from unity. This procedure was done above.

In dimensionless variables the equations retain their shape. Therefore, when writing algorithms and programs, can use the original form of the dimensional equations and dimensionless variables, used in the calculations by specifying the input data, for the coefficients of the equations and boundary conditions, in accordance with the variant of nondimensionalization variables. The data show that with the increase of the order, decrease in the concentration of the reagent with time becomes less intense.

Based on these kinetic curves for the particles in the process of gelation in the reaction of zero order, with $n=0$, and the rate constant is corresponds to the frequency of collisions. Hence, using equation (4.127) and (4.128) for the model in the framework of statistical thermodynamics (Section 4.9), for the dimensionless parameters we have degree of conversion of primary particles of 0.5 . Accordingly, the dimensionless concentration will also be equal to 0.5 . As a result, we obtain the dimensionless gel time for the cases of processes with different order of the reaction. The results are shown in Figures 16 and 17, and in Table 2.

Table 2. Dimensionless time for the gelation process, with a different order of the reaction.

\begin{tabular}{|c|c|c|c|c|}
\hline \multirow{2}{*}{} & \multicolumn{4}{|c|}{ The number of particles in the elementary act } \\
\cline { 2 - 5 } & $n=0$ & $n=1$ & $n=2$ & $n=3$ \\
\hline $\boldsymbol{t}_{\boldsymbol{c}}$ & 0.500000 & 0.693147 & 1.000000 & 1.500000 \\
\hline
\end{tabular}

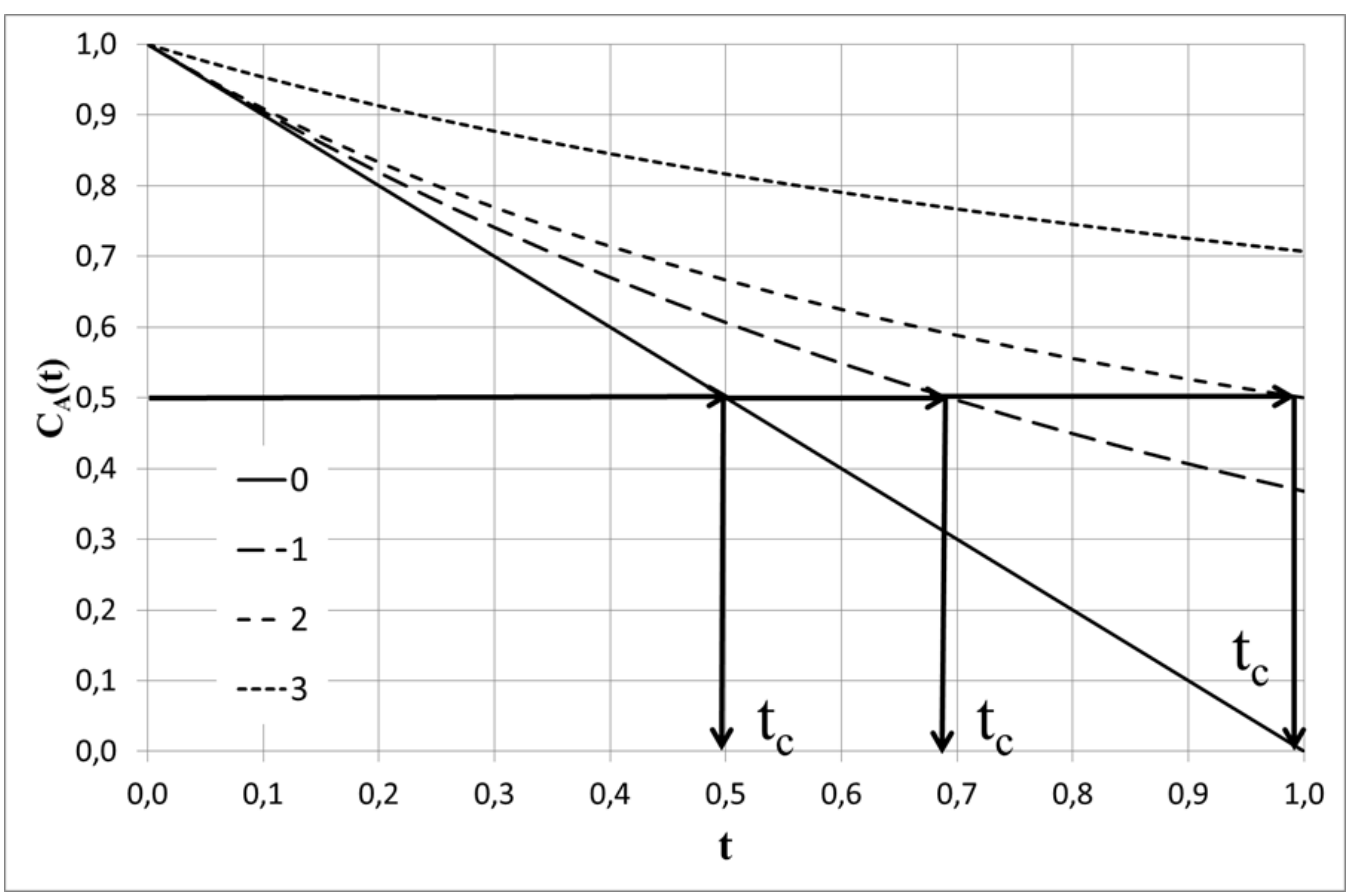

Figure 16. The behavior of the kinetic curves of consumption of the sol particles in the elementary reactions of various orders. 
$\boldsymbol{k}=1 ; C_{A}(0)=1 ; \boldsymbol{t}_{\boldsymbol{c}}$ - the dimensionless time of gelation. The reaction order: $0 ; 1 ; 2 ; 3$.

Considering the elementary act of interaction of particles sols each other, we can conclude that the case of the reaction order $n=0$ and $n=1$, have more likely asymptotic and the theoretical value. Since their physical meaning in this case is not clear enough. However, a deeper physical meaning bear in themselves model of the processes of the second and third orders. This is due to the fact that the second-order reaction is the interaction of two identical particles with each other, and the third order - the simultaneous collision of three particles. Naturally the probability of the second process is significantly lower. Such processes will likely determine the appearance of particles with a coordination number of 3 or more in the gel structure. They will also lead to an increase of the fractal dimension of the emerging gel, causing increase of its $D_{f}>2$. Furthermore, from the data in Figure 16 and Table 2, it is seen that the order of reaction, which is equal to 2 , the speed of interaction, and thus the dimensionless time to gelation is reduced 2 times in comparison with the case of only the probability of collision frequency.

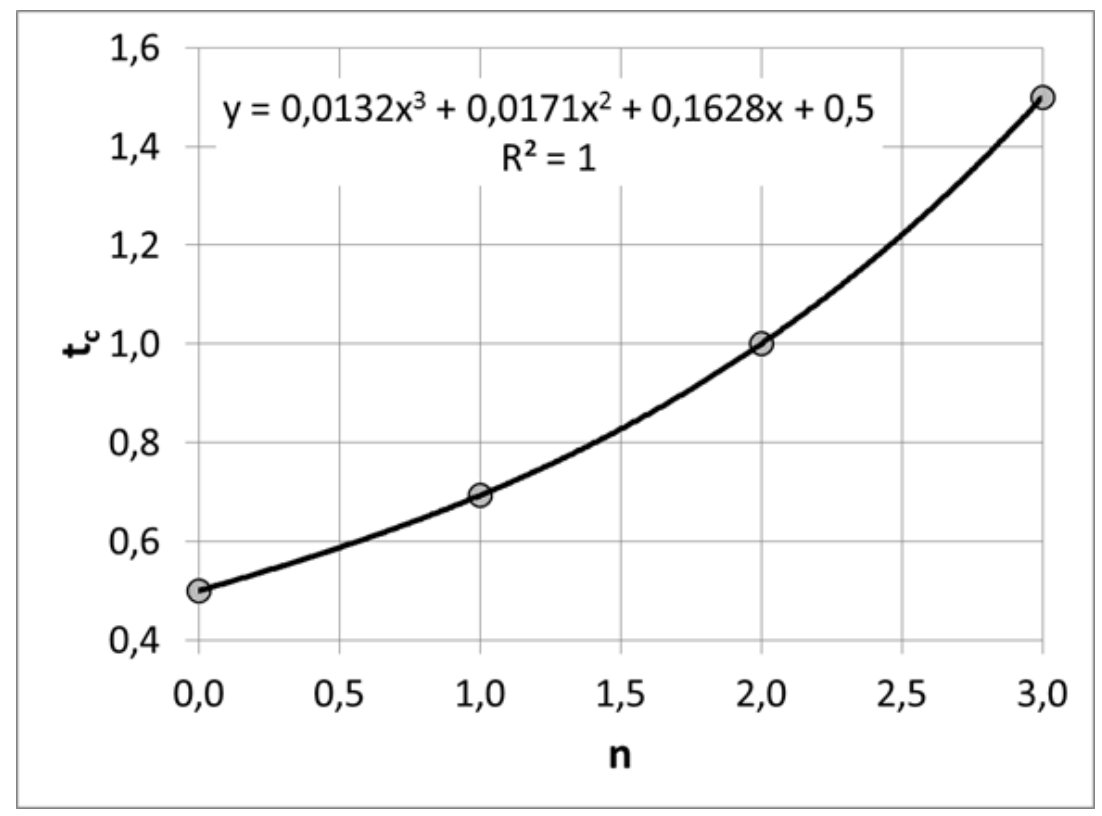

Figure 17. Dependence of the dimensionless time of gelation from the reaction order.

As seen in Figure 17, the dependence of the dimensionless time of gelation from the reaction order is nonlinear and is well approximated by a polynomial of degree 3 . This once again confirms the probabilistic nature of the interaction of the sol particles, with each other, in the process of gelation.

\section{CONCLUSION}

The statistical polymer method is based on the consideration of averaged structures of all possible macromolecules of the same weight. One has derived equations allowing evaluation of 
all additive parameters of macromolecules and their systems. The statistical polymer method allows modeling of branched crosslinked macromolecules and their systems in equilibrium or non-equilibrium. The fractal consideration of statistical polymer allows modeling of all kinds of random fractal and other objects studied by fractal theory. The statistical polymer method is applicable not only to polymers but also composites, gels, assonates in polar liquids and other aggregates. Analysis of the behavior of silica sol, in terms of Maxwell-Boltzmann distribution allowed calculating the mean free path of the colloidal particles. Based on these data, it was calculated the number of particles capable to overcome the potential barrier in a collision. To modeling of the sol-gel transition kinetics had considered various approaches.

\section{References}

[1] Sletteri J., Theory of transfer of momentum, energy and mass in continuous media, M., Mir, (1978).

[2 ] Heifetz L.I., Neumark A.V., Multiphase processes in porous media, M., (1982).

[3] Greiser, T., Jarchow, O., Klaska, K.-H. and Weiss, E. (1978), Dioxotetradecakis(trimethylsiloxo)octadecakupfer(I), Cu18O2[OSi(CH3)3]14, ein silikon-analoges Oligomeres mit Kupfer als Heteroatom im silikatischen Grundgerüst. Chem. Ber., 111: 3360-3366. doi: 10.1002/cber.19781111010

[4] Frank-Kamenetsky D.A., Diffusion and Heat Transfer in Chemical Kinetics, 3rd ed., M., (1987).

[5] Heifetz L.I., Bruno E.B., Theoretical Foundations of Chemical Engineering 21(2) (1987) 191-214.

[6] Flory, P.J. Statistical Mechanics of Chain Molecules. Interscience, New York, (1969).

[7] Moshinsky, L. and Figovsky, O. Proc. Intern. Conf. "Corrosion in Natural and Industrial Environments: Problems and Solutions”, (1995), 699 p.

[8] Romm F. Derivation of the Equations for Isotherm Curves of Adsorption on Microporous Gel Materials, Langmuir, (1996), 12, 14, pp. 3490-3497.

[9] Romm F., J. Phys. Chem. 98(22) (1994) 5765-5767.

[10] Gontar, V. New theoretical approach for physicochemical reactions dynamics with chaotic behaviour. In Chaos in Chemistry and Biochemistry, World Scientific, London, 1993, pp. 225-247.

[11] Morachevsky A.P. Physical chemistry - surface phenomena and disperse systems - SPb., 2011.

[12] Smoluchowski M.V., Zeitschrift Fur Physikalische Chemie 92 (1917) 129.

[13] Schuman T.E.W., Quart. Yourn. R. Met. Soc. 66 (1940) 195.

[14] Ziff R. M. G., J. Chem. Phys. 73(7) (1980) 3492.

[15] Vinokourov L.I., Kats A.V. Power solutions of the kinetic equation for fixed aerosol coagulation. Izvestiya Academy of Sciences of the USSR. Physics of the atmosphere and ocean. 16(6) (1980) 601-607. 
[16] Stockmayer W.H.Y. Chem. Phys. 11 (1943) 45.

[17] White W.H.Y. Colloid Interface Sci. 87 (1982) 204.

[18] Lushnikov A.A. Some new aspects of the theory of coagulation. Izvestiya Academy of Sciences of the USSR. Physics of the atmosphere and ocean. 14(10) (1978) 1046-1054.

[19] Domilovsky E.R., Lushnikov A.A., Piskunov V.N. Modeling of coagulation process Monte Carlo. DAN, 240(1) (1978) 108-110.

[20] Bondarev B.V., Kalashnikov N.P., Spirin G.G. General physics course: in 3 books. Book 3. Statistical physics. Structure of Matter. M.: Yurayt, 2013, 369 p.

[21] Zhyulne R. Fractal aggregates, UFN, 157(2) (1989) 339-357.

[22] Smirnov B.M. Properties of fractal aggregate, UFN, 157(2) (1989) 357-360.

[23] Lifshitz, EM, Pitaevskii, LP Statistical physics. Part 2. The theory of the condensed state. ("Theoretical Physics", Vol IX). - M.: FIZMATLIT, 2004. - 496 p.

[24] Levenshpil O. Engineering design of chemical processes, Chemistry, 1969

[25] Dorohov I.N., Kafarov V.V. Systematic analysis of the processes of chemical technology. Nauka, Moscow, 1989, 376 p. 